Meta

Journal des traducteurs

Translators' Journal

\title{
La recherche traductologique dans les domaines de spécialité : un nouveau tournant
}

\section{Sylvie Vandaele}

Volume 60, numéro 2, août 2015

$60^{\mathrm{e}}$ anniversaire. Les horizons de la traduction : retour vers le futur $60^{\text {th }}$ Anniversary. Translation's Horizons: Back to the Future

60mo aniversario. Los horizontes de la traducción: regreso al futuro

URI : https://id.erudit.org/iderudit/1032855ar

DOI : https://doi.org/10.7202/1032855ar

Aller au sommaire du numéro

Éditeur(s)

Les Presses de l’Université de Montréal

ISSN

0026-0452 (imprimé)

1492-1421 (numérique)

Découvrir la revue

Citer cet article

Vandaele, S. (2015). La recherche traductologique dans les domaines de spécialité : un nouveau tournant. Meta,60(2), 209-237.

https://doi.org/10.7202/1032855ar
Résumé de l'article

La recherche liée à la traduction pragmatique, et notamment dans les domaines de spécialité est, historiquement, minoritaire, car la traductologie occidentale s'est essentiellement développée à partir de questions soulevées par la traduction littéraire. Pourtant, la traduction pragmatique domine largement la pratique professionnelle. De même, la recherche traductologique a été l'objet de réticences de la part des praticiens et des étudiants. Nous formulons l'hypothèse que les préjugés négatifs, des deux côtés, trouvent leur source chez certains auteurs princeps fondateurs de la traductologie. La présente conférence explore ainsi les positions exprimées chez Berman, Schleiermacher et Ortega y Gasset, et remet en question la carte de Holmes, pour arriver à proposer une autre carte qui ouvre toutes les possibilités autant sur le plan de la recherche que sur le plan de la pratique. Par ailleurs, en raison du développement des outils informatiques, nous sommes convaincue que le traducteur devra de plus en plus se positionner comme un expert capable de conseiller adéquatement les donneurs d'ouvrages. Nous nous proposons donc, pour terminer, de dégager quelques pistes de recherche, concernant plus spécifiquement les domaines de spécialité, qui pourront s'épanouir dans le contexte de la traductologie, avec pour arrière-plan une volonté délibérée d'arrimer la pratique à la recherche et à la réflexion dite " théorique », et réciproquement.
Ce document est protégé par la loi sur le droit d'auteur. L'utilisation des services d'Érudit (y compris la reproduction) est assujettie à sa politique d'utilisation que vous pouvez consulter en ligne.

https://apropos.erudit.org/fr/usagers/politique-dutilisation/ 


\title{
La recherche traductologique dans les domaines de spécialité: un nouveau tournant
}

\author{
Sylvie Vandaele \\ Université de Montréal, Montréal, Canada \\ sylvie.vandaele@umontreal.ca
}

\section{RÉSUMÉ}

La recherche liée à la traduction pragmatique, et notamment dans les domaines de spécialité est, historiquement, minoritaire, car la traductologie occidentale s'est essentiellement développée à partir de questions soulevées par la traduction littéraire. Pourtant, la traduction pragmatique domine largement la pratique professionnelle. De même, la recherche traductologique a été l'objet de réticences de la part des praticiens et des étudiants. Nous formulons l'hypothèse que les préjugés négatifs, des deux côtés, trouvent leur source chez certains auteurs princeps fondateurs de la traductologie. La présente conférence explore ainsi les positions exprimées chez Berman, Schleiermacher et Ortega y Gasset, et remet en question la carte de Holmes, pour arriver à proposer une autre carte qui ouvre toutes les possibilités autant sur le plan de la recherche que sur le plan de la pratique. Par ailleurs, en raison du développement des outils informatiques, nous sommes convaincue que le traducteur devra de plus en plus se positionner comme un expert capable de conseiller adéquatement les donneurs d'ouvrages. Nous nous proposons donc, pour terminer, de dégager quelques pistes de recherche, concernant plus spécifiquement les domaines de spécialité, qui pourront s'épanouir dans le contexte de la traductologie, avec pour arrière-plan une volonté délibérée d'arrimer la pratique à la recherche et à la réflexion dite «théorique», et réciproquement.

\begin{abstract}
Historically, research on pragmatic translation, namely in the case of specialized fields, has been neglected, since Western translation studies were mainly established around questions pertaining to literary translation. Yet, pragmatic translation is largely dominant in professional practice. Similarly, professional translators and translation students have been reluctant to engage with translation studies research. We hypothesize that these negative prejudices, on both sides, originate with some of the founding, seminal authors of translation studies. This conference thus explores the theoretical positions expressed by Berman, Schleiermacher, and Ortega y Gasset; it also questions Holmes' map, before proposing an alternative map, one that opens all possibilities, from the point of both research and practice. Moreover, given the development of computer tools, we are convinced that translators will increasingly have to present themselves as experts capable of offering adequate guidance to clients. Finally, we propose to highlight a number of research perspectives, related more specifically to specialised fields, which can blossom in the context of translation studies, keeping in mind our deliberate intent to reconcile translation practice with translation research and so-called "theory," and vice versa.
\end{abstract}

\section{RESUMEN}

Aunque la traducción pragmática domina ampliamente la práctica profesional, la investigación ligada a la traducción pragmática, y más precisamente a las áreas de especialidad ha sido, históricamente, minoritaria, ya que la traductología occidental se desarrolló esencialmente a partir de interrogantes generados por la traducción literaria. Así mismo, la investigación traductológica ha generado reticencia en los profesionales y los estudian- 
tes. Nuestra hipótesis es que estos prejuicios negativos tienen su origen en algunos autores primigenios fundadores de la traductología. La presente conferencia explora las posiciones expresadas por Berman, Schleiermacher y Ortega y Gasset, y cuestiona el esquema propuesto por Holmes, para así llegar a la propuesta de otro esquema que abre todas las posibilidades tanto en el plano de la investigación como en el de la práctica. Además, como resultado de la evolución de las herramientas informáticas, afirmamos que el traductor está cada vez más llamado a posicionarse como un experto capaz de aconsejar adecuadamente a su cliente. Nos proponemos entonces, para concluir, ofrecer algunas pistas de investigación, específicamente relacionadas con las áreas de especialidad, que pueden germinar en el contexto de la traductología, teniendo como trasfondo una voluntad deliberada de acercar la práctica a la investigación y a la reflexión llamada "teórica" y viceversa.

\section{MOTS-CLÉS/ KEYWORDS/PALABRAS CLAVE}

traduction pragmatique, domaine de spécialité, recherche, théorie, pratique pragmatic translation, specialty domain, research, theory, practice traducción pragmática, campo de especialidad, investigación, teoría, práctica

We need to be provoked, challenged, contested [...].

(Bassnett 2012: 22)

Rien n'a changé [pour la traduction] dans la mesure où on continue de traduire sans prendre les acquis éventuels de la traductologie. [...] Tout a changé pour la traductologie tout simplement parce qu'elle est née.

(Nouss, 2012, in Basalamah 2012: 30)

\section{Introduction}

À l'affirmation provocatrice de Nouss placée en exergue, on peut opposer le témoignage de traductrices et de traducteurs dont la pratique s'inspire de la réflexion traductologique, comme celui de Godard (2001), et qui ont suivi l'appel lancé par Berman dans L'épreuve de l'étranger (1985: 300). Mais, de fait, combien sont-ils? L'intimité cognitive de l'acte traductionnel ne permet pas d'en révéler les allégeances. Nouss n'est ni le seul ni le premier à déplorer le fossé entre la théorie et la pratique (voir par ex. Bensimon 1987). Évoque-t-il strictement la pratique de la traduction littéraire ou étend-il son constat à l'ensemble de l'activité traduisante et notamment à celle qui se pratique chaque jour dans les cabinets, les services de traduction ou les microentreprises (travailleurs autonomes), c'est-à-dire ce que l'on appelle généralement "traduction pragmatique $»^{1}$ ? Les praticiens ne se sentent généralement pas concernés par la théorie, et les étudiants la trouvent généralement trop abstraite et préfèrent "passer à l'acte» au plus vite.

Le fait est que ce qui est nommé théorie en traductologie s'est forgé essentiellement à partir de la littérature, tout au moins en Occident: son influence sur la traduction pragmatique reste donc pratiquement inexistante, sinon nulle. Pourtant, selon Vande Walle (2007, cité par Scarpa 2010: 85; traduit par Fiola), «en 2006 en Europe, $99 \%$ des textes traduits étaient des textes pragmatiques», et plus particulièrement dans les domaines de spécialités (technique, commercial, juridique, médical, administratif et scientifique). Au Canada, et plus précisément au Québec, les lois linguistiques commandent la prédominance de ce type de traduction. 
Il existe une recherche traductologique portant sur la «non-littérature», mais elle reste minoritaire (Aixelá 2004). Selon Vande Walle (2007), $1 \%$ des textes traduits sont destinés au grand public: par conséquent, l'accessibilité aux textes par le chercheur est rendue beaucoup plus difficile que pour la littérature, ce qui peut expliquer en partie le manque de recherche. Toutefois, la visibilité, par Internet, de documents autrefois inaccessibles permet d'envisager de combler partiellement ce vide. En ce qui concerne les travaux qui s'intéressent aux agents, la dissémination de la pratique liée à l'usage des communications électroniques ne rend pas très facile la mise en œuvre d'études de terrain. Par ailleurs, historiquement, la recherche dans les domaines de spécialité s'est surtout concentrée sur la terminologie, mais comme Rogers le souligne, "terminological knowledge is a necessary but insufficient condition for specialised translators to do their job» (Rogers 2015: 3). Enfin, les recherches dans le secteur des «langues de spécialité» (Language for Specific Purposes, LSP) sont en grande partie à portée unilingue. Pourtant, Froeliger, se faisant l'avocat du développement d'une traductologie pragmatique, affirme: «Est-il normal, est-il sain, est-il souhaitable que le gros de la recherche en traduction se concentre encore dans la sphère littéraire alors que la grande majorité des traductions s'effectuent dans le domaine pragmatique? Non.» (Froeliger 2010: 655)

Il se trouve que certains traductologues, depuis quelques années, s'interrogent. Ainsi, Bassnett, après avoir évoqué les débuts hérö̈ques et enthousiasmants de la traductologie $e^{2}$, aspire à un renouveau (Bassnett 2012). Pour sa part, Pym plaide pour une remise en question «des deux côtés de l'Atlantique», en affirmant que les changements viennent davantage de la pratique professionnelle que de la traductologie, et notamment de l'arrivée des outils informatiques que de la traductologie (Pym 2009: 69). Faut-il conclure à un relatif échec de la traductologie? Sûrement pas: outre la mise en place de nombreux programmes d'enseignement qui forment maintenant des doctorants et de futurs chercheurs (voir par ex. Kelly 2005 et l'article de Hurtado dans le présent volume), on ne peut que constater le dynamisme de la discipline par la prolifération des publications (plus de 110 revues au monde, plus de 60000 publications, monographies, encyclopédies, compilations...) et des colloques (Munday 2012: empl. 547³ ; Rovira-Esteva, Orero et Aixelá 2015: 159).

Partons donc de cet état des lieux: la traductologie est née, elle s'est construite à partir de la littérature, elle peine cependant à se faire entendre des praticiens; malgré l'apparition et le développement de nouveaux sujets de recherche, beaucoup reste à faire, et notamment en rapport avec la traduction pragmatique. Et tentons de comprendre les racines de cette fracture pour mieux orienter l'avenir. Dans le présent article, nous commencerons par nous interroger sur certains des fondements de la traductologie, qui, s'ils ont sans conteste une valeur historique et fondatrice, pourraient l'avoir orientée dans une impasse. Celle-ci pourrait peut-être expliquer la tension entre traduction littéraire et traduction pragmatique, qui n'a, à notre avis, pas lieu d'exister. Il n'entre d'ailleurs pas dans notre intention de nier la valeur du littéraire, nous y sommes au contraire fort attachée. Nous prendrons ensuite le risque de revisiter le rapport établi par Holmes entre la traductologie (ou plutôt, en ce qui le concerne, le concept de translation studies) et les sciences empiriques, ainsi que sa cartographie de la discipline. Ceci nous mènera à proposer une nouvelle carte de la traductologie, centrée sur l'acte traductionnel, qui permet d'ouvrir, conceptuellement, des perspectives favorisant le dialogue entre recherche, pratique et enseignement. Enfin, nous proposerons pour conclure quelques pistes de recherche. 


\section{Vérité, pureté, vraie langue: Berman, Schleiermacher et Ortega y Gasset}

À la recherche des principes fondant une traductologie excluant la traduction pragmatique, nous avons suivi un fil d'Ariane qui passe par la filiation intellectuelle d'Antoine Berman (1942-1991) avec Friedrich Daniel Ernst Schleiermacher (17681834). Les questions d'intraduisibilité, de prééminence des cultures et d'écart entre la traduction littéraire ou savante et la traduction pragmatique les caractérisent. Foz évoque la proximité entre la «folle entreprise» qu'est la traduction pour Schleiermacher et l'utopie de José Ortega y Gasset (1883-1955) (Foz 2004: 14), qui lui aussi manifeste un rejet de toute forme non littéraire de la traduction. Bien que de filiations philosophiques distinctes ainsi que de pays et d'époques relativement distants, Schleiermacher et Ortega y Gasset sont des références marquantes en traductologie et méritent donc que l'on s'y intéresse si l'on veut comprendre les tensions qui déchirent la discipline.

\subsection{De la vraie traduction: Berman}

Un texte technique (si tant est qu'on puisse ici parler de texte)...

(Berman 1985b: 84)

La lecture de textes fondamentaux de la traductologie tels que ceux de Berman peut se révéler troublante pour un traducteur œuvrant déjà, ou se destinant à œuvrer, en traduction pragmatique: dès sa formation, le traducteur en herbe se voit relégué du bord d'un utilitarisme somme toute peu glorieux. Pour Berman, l'opposition entre l'œuvre littéraire et le "texte technique» (entendu au sens large) relève de leurs fonctions respectives: contrairement au texte technique, l'œuvre n'a pas pour fonction de communiquer quoi que ce soit (Berman 1985b: 84); et donc, elle ne relève pas d'une quelconque méthodologie qui ne s'appliquerait qu'à « un processus de communication, de transmission de "messages" d'une langue de départ (dite langue-source) à une langue d'arrivée (dite langue-cible)»:

Un texte technique (si tant est qu'on puisse ici parler de texte) est certainement un message visant à transmettre de manière (relativement) univoque une certaine quantité d'informations; mais une ouvre ne transmet aucune espèce d'information, même si elle en contient: elle ouvre à l'expérience d'un monde. C'est donc seulement à un niveau d'abstraction très élevé qu'on peut leur trouver un dénominateur commun, au prix d'amalgamer les messages et les textes. Les messages, assurément, relèvent d'une méthodologie; les textes, sûrement pas. (Berman 1985b: 84; nous soulignons)

Berman se défend de hiérarchiser l'un par rapport à l'autre, mais la nature de son propos le fait de facto, et notamment dans la conclusion, lourde de conséquences:

Il ne s'agit pas, ici, d'établir une hiérarchie, mais de différencier définitivement les domaines: un texte n'est jamais un message, et vice-versa. Cela ne veut pas dire non plus que les œuvres ne relèvent que d'un pur artisanat intuitif. Leur traduction, au contraire, exige une haute systématicité: mais système n'est pas méthode. Les principes qui régissent la systématique de la traduction des œuvres relèvent d'une certaine visée, et cette traduction ne sera communication que si, a priori, elle se soumet à un impératif plus haut que toute communication. Du reste, chaque fois que la traduction dite "littéraire" se pose comme acte de communication, elle devient inévitablement noncommunication. Bref, le concept de communication est pour nous trop abstrait pour définir l'œuvre et sa traduction. C'est un concept que la technologie a confisqué définiti- 
vement, et on peut le lui laisser. Il appartient à la traductique, non à la traductologie. (Berman 1985b: 84; nous soulignons)

La caractérisation de l'œuvre comme « ouvrant à l'expérience d'un monde» n’est guère réfutable. La grandeur de l'œuvre tient en fait à cette possibilité d'expérience, mais elle n'en a pas l'exclusivité. Par les conceptualisations qu'il recèle, le texte pragmatique est aussi expérience d'un monde (voir par ex. les conceptualisations en biologie, Vandaele 2009). Ce qui laisse rêveur, c'est la radicalité avec laquelle Berman renvoie le texte technique à une «simple» communication, à la fois trop abstraite et relevant d'une mécanique assimilée à la traductique. Nous sommes devant un univers dichotomisé, manichéen, qui ne rend pas compte de la diversité, de la complexité et du rôle socialement essentiel de la traduction pragmatique. La métaphorisation de la verticalité ramène nécessairement le lecteur à l'idée que LE MIEUX EST EN HAUT (comme l'écriraient Lakoff et Johnson, 1985/2003), c'est-à-dire selon une métaphore conceptuelle organisant subtilement la dominance du littéraire par rapport au nonlittéraire.

La même dichotomie hiérarchisante se retrouve dans d'autres écrits, cette fois-ci élargie à tout ce qui est «non littéraire»:

Cette distinction, ne correspond-elle pas simplement à cette grande coupure qui divise tout le champ de la traduction, celle qui sépare les traductions dites «littéraires» (au sens large) des traductions «non littéraires» (techniques, scientifiques, publicitaires, etc.)? Alors que ces dernières n'opèrent que des transferts de sens et que les textes auxquels elles ont affaire entretiennent un rapport d'extériorité ou d'instrumentalité avec leur langue, les premières s'occupent d'œuvres, soit de textes tellement liés à la langue que l'acte de traduire devient ici fatalement un travail sur les signifiants, un travail où, selon des modes variables, deux langues entrent en commotion, et d'une certaine manière s'accouplent. (Berman 1985a: 68; nous soulignons)

Ladmiral, dans un hommage posthume à celui qu'il appelle «mon ami personnel et mon adversaire théorique privilégié» rappelle que Berman fut pourtant, à ses heures, un traducteur technique (Ladmiral 1992: 63). Si l'on se fie à ses écrits, il dut en souffrir et ne pas y trouver l'exutoire réclamé par son idéalisme. L'assertion selon laquelle le texte non littéraire est hors de la langue, ou dans un rapport strict d'instrumentalité excluant tout travail sur le sens va totalement à l'encontre de notre position.

\subsection{De la pureté des écrits académiques: Schleiermacher}

Remonter le fil suivi par Berman permet de comprendre, notamment chez Schleiermacher, un des nœuds fondant cette rupture et cette hiérarchisation. Rappelons que Schleiermacher, théologien protestant et philosophe, fonde l'herméneutique en tant que courant philosophique et influence fortement certains traductologues tels que Benjamin, Berman et plus tard Venuti (Lianeri 2002). Son ouvrage Ueber die verschiedenen Methoden des Uebersezens (Schleiermacher 1813/1838, traduit par Berman en 1985 (Des différentes méthodes du traduire [Schleiermacher 1813/1985/1999]), par Lefevere en anglais (On the Different Methods of Translating, dans Lefevere 1977), et par García Yebra en espagnol (Sobre los diferentes métodos de traducir [Schleiermacher 1978/2000]) recèle les préceptes de celui qui fut un représentant actif du romantisme allemand. Ce courant est généralement caractérisé par son opposition aux Lumières et à leur rationalité cartésienne, laquelle préfigure le positivisme de la fin du XIX ${ }^{\mathrm{e}}$ siècle 
(Lianeri 2002). Bien que ce rejet des Lumières ne semble pas aussi radical qu'on l'affirme généralement, "[l]e motif ordinaire de cette critique est l'imputation d'universalisme abstrait, de pensée mécanique, réduite au calcul, d'ignorance des particularités, souvent de grossier matérialisme et d'empirisme.» (Thouard 2003: s.p.). Thouard précise que Schleiermacher s'en prenait «à la rage de comprendre» imputable aux Lumières et incarnée particulièrement par les Anglais et les Français:

Cette promotion de la raison relativise toute chose, qui perd son individualité, dès lors qu'elle est comprise dans un contexte abstrait, celui de la nature soumise à des lois, ignorant toute particularité:

"On exige de tout un dessein, un but; chacun doit toujours accomplir quelque chose [...] leur grand but, c'est la place qu'un objet prend dans la série des phénomènes." (Thouard 2003, citant Schleiermacher 1799/1944: 148-150)

Dans un tel contexte où la rationalité est contestée, la traduction que Berman fait de Schleiermacher soulève quelques questions. Selon cette traduction, Schleiermacher établit la véritable traduction "dans le domaine de la science et de l'art» (in dem Gebiete der Wissenschaft und Kunst) (Schleiermacher 1813/1985/1999: 32-35; traduction par Berman) ${ }^{4}$. La traduction écrite est ramenée à un "enregistrement d'une transposition orale» (Aufzeichnung einer mündlichen anzusehen), car seul n'est envisagé, en dehors du monde de la «science et de l'art», que celui des "affaires» (Geschäften), où «l'écriture n'est qu'un moyen mécanique» (ist die Schrift nur mechanisches Mittel), et «le commerce oral», «primordial» (das mündliche Verhandeln ist darin das ursprüngliche). Or, en ce début de $\mathrm{XIX}^{\mathrm{e}}$ siècle, le terme allemand Wissenschaft ne réfère pas à la science héritée des Lumières:

In the early nineteenth century, German natural researchers joined other educated men in celebrating the ideal of pure Wissenschaft, which was deeply disdainful of enlightened utilitarianism. (Philips 2012: 87)

In championing the cause of pure Wissenschaft, leading natural researchers evoked images of a virtuous but declining learned estate, threatened by masses of second-rate, petty, and materialistic laborers. [...] The positive adjectives that Enlightenment societies had often affixed to natural knowledge, words like "useful" or "public spirited," were the particular object of scorn. (Philips 2012: 100)

If the university passed on Wissenschaft to the next generation, the society or academy was the place where the accomplished researcher met his peers. These were the forums in which "the masters of Wissenschaft [came] together," to quote Friedrich Schleiermacher. (Philips 2012: 99)

Selon Philips, le romantisme mène à l'effritement de l'alliance «théorie et pratique» "des sciences et des arts» (Wissenschaften und Künsten): sous l'influence des Lumières ainsi que de leur vision de l'universalité et de l'utilité des connaissances, la philosophie naturelle et l'histoire naturelle faisaient l'objet de débats dans le même cadre que l'amélioration de l'agriculture. Mais cette alliance perdit du terrain lorsque la critique des Lumières se fit dominante: "by the early nineteenth century, this unifying rubric had lost a great deal of ground.» (Philips 2012: 98)

Ladmiral, traducteur de la philosophie allemande, explique que Wissenschaft a un sens bien plus large que le français science dans son sens actuel,

tellement plus large qu'on peut presque parler d'un sens différent. En français, il s'agit d'un concept bien précis qui désigne un savoir cumulatif et structuré, satisfaisant aux 
exigences de la méthode expérimentale et de la formalisation logico-mathématique; c'est une catégorie épistémologique. En allemand, est Wissenschaft tout savoir ayant une méthodologie propre et définie, et de fait tout savoir enraciné dans l'institution universitaire; c'est plutôt une catégorie historique ou socioculturelle, voire une catégorie socioprofessionnelle. (Ladmiral 2002: 107)

Ce à quoi Schleiermacher réfère par Wissenschaft correspond davantage à l'activité universitaire, donc aux écrits savants, qu'à la science telle que nous pouvons la concevoir de nos jours. De manière intéressante, si Berman en français (Schleiermacher 1813/1985/1999: 33) et García Yebra en espagnol (Schleiermacher 1978/2000: 227) ont traduit Wissenschaft par science et ciencia, respectivement ${ }^{5}$, Lefevere, en anglais, l'a traduit par scholarship (Lefevere 1977: 68), et non par science. Pour comprendre la pensée de Schleiermacher, et ne pas y voir de contradiction interne, il est donc fondamental de comprendre ce cas de... quasi-intraduisibilité! Par ailleurs, cette tension renvoie également à celle que nous évoquerons plus loin, entre le savoir "pur» et «appliqué».

\subsection{La pseudolangue de la science: Ortega y Gasset}

Ortega y Gasset, philosophe, homme politique et journaliste, "penseur fondamentalement européen» et, selon Wuillème (2011), beaucoup moins conservateur qu'on a pu le dire, s'intéresse lui aussi à la traduction. L'écart entre traduction littéraire et traduction pragmatique paraît chez lui insurmontable. Ortega y Gasset explique la «facilité» avec laquelle certains ouvrages scientifiques peuvent, selon lui, être traduits par l'existence d'une «pseudolangue» dans laquelle l'auteur «se traduit lui-même» à partir «de la langue authentique». Cette pseudolangue est "composée de termes techniques, de vocables linguistiquement artificiels dont il [l'auteur] doit lui-même donner la définition». L'incompréhension des notions scientifiques - non évoquées en tant que telles - est ainsi ramenée à l'ignorance d'une langue artifielle, la langue commune étant la véritable langue, celle qui

[...] est un système de signes verbaux grâce auxquel les hommes peuvent se comprendre sans accord préalable alors qu'une terminologie n'est intelligible que dans la mesure où celui qui l'emploie à l'écrit ou à l'oral s'est préalablement entendu individuellement avec son lecteur ou son auditeur sur le sens des signes. C'est pourquoi je l'appelle pseudolangue et dis que l'homme de science doit d'abord traduire sa propre pensée dans cette langue. C'est un volapük, un espéranto délibérément établi entre les spécialistes d'une discipline. D'où la relative facilité à faire passer ce genre d'ouvrages d'une langue à une autre. Car d'où qu'ils viennent, ils sont déjà presque entièrement écrits dans la même langue. À telle enseigne qu'ils semblent hermétiques, inintelligibles ou à tout le moins très ardus à tous ceux qui parlent la langue authentique dans laquelle ils sont apparemment écrits. (Ortega Y Gasset 1937/2004: 18-21; traduit par Clara Foz) ${ }^{6}$

L'ironie est que, quelques lignes plus loin, Ortega y Gasset illustre (tout en contournant la nécessité d'une démonstration rigoureuse: "Je vous fais grâce du couplet») l'intraduisibilité de la littérature par ce qui se révèle, au fond, un banal problème de terminologie auquel se heurte quotidiennement tout traducteur, littéraire ou non:

Les langues s'étant formées dans des environnements différents et par des expériences diverses, il est naturel qu'elles ne coïncident pas. Il est faux, par exemple, de penser que ce qu'un Espagnol appelle bosque correspond à ce qu'un Allemand appelle Wald, et 
pourtant le dictionnaire indique que Wald signifie bosque. Si le cœur y était, quelle excellente occasion ce serait d'y aller d'un morceau de bravoure sur tout ce qui sépare un bois allemand d'un bois espagnol. Je vous fais grâce du couplet, mais en revendique le résultat, soit la claire intuition de l'énorme différence existant entre ces deux réalités.

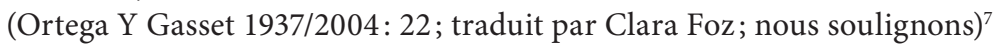

Il ne s'agit pas de remettre en cause l'intérêt indéniable, historique, des écrits de figures marquantes comme Ortega y Gasset, mais le risque, si l'on s'y attache sans replacer la réflexion dans son contexte, est la sclérose de la pensée traductologique. Si l'on poursuit son raisonnement jusqu'au bout, la langue «vraie», celle grâce à laquelle «les hommes peuvent se comprendre sans accord préalable» serait alors amalgamée avec la pensée: le rapport signifiant-signifié n'existe plus! Il va de soi que, depuis Ortega y Gasset, les travaux de linguistique, de terminologie et de sémantique référentielle sont autant de réfutations d'une telle position. Les langues n'existent pour leurs locuteurs que dans la mesure où ils les ont apprises. Les concepts d'intersubjectivité partagée (Kleiber 2001) et de construction dynamique du sens (Victorri 1994) permettent d'expliquer la construction des langues et des normes linguistiques tout autant que leur variabilité et leur évolution. La différence entre le bois espagnol et le bois allemand relève d'un problème de prototypie du sens et de représentation du monde que les approches cognitives ont amplement discuté. Quant à l'existence d'une langue scientifique, le fait que l'on parle couramment de langue de spécialité (Language for Specific Purposes) pourrait nous abuser. Un discours scientifique, ou technique, ou plus largement, spécialisé, comporte certes des particularités (style, normes, vocabulaire), mais il s'inscrit dans une langue donnée (anglais, français, etc.), il se soumet aux règles générales de la langue même s'il en déroge parfois. Le vocabulaire scientifique n'est de plus pas disjoint d'une langue qui serait dite générale - comme en témoigne notamment le passage de termes spécialisés dans les communications quotidiennes. À l'inverse, nous avons, nous-même, souligné plusieurs fois les difficultés importantes liées à la traduction d’unités terminologiques spécialisées qui ont une "apparence» (le signifiant) des plus banales (Vandaele 2006a; 2006b; 2008), notamment certaines unités prédicatives (par exemple, inhibition, stimulation, etc. voir Vandaele et Raffo 2008). Parler de langue de spécialité et de langue générale est pour nous une fiction opératoire, utile pour délimiter des pans de programme, des regroupements d'intérêts de recherche, voire des nomenclatures de dictionnaire, mais trompeuse si l'on n'y prend pas garde: le vocabulaire de la médecine ou de toute autre spécialité ne constitue pas une langue étrangère! Enfin, il suffit de pratiquer la traduction spécialisée pour réfuter l'idée que les textes spécialisés relèvent d'une mécanique, tant ils exigent des compétences avancées, et ce, même si, comme le concède Froeliger (2010), les possibilités de traduction sont moins ouvertes qu'en littérature.

Quant à savoir si la traductique pourra faire aussi bien ou mieux que l'humain, cela sera l'enjeu d'un futur relativement proche: ce qui est certain, c'est que si les approches statistiques de la traduction automatisée permettent d'obtenir des résultats parfois étonnants, elles demeurent impuissantes dès lors que les textes à traduire sont mal représentés dans les corpus d'analyse. 


\section{La carte de la traductologie}

In 2015 a scholar in TS cannot ever hope to cope with the more than 3000 new publications issued each year. We need maps, and we need to know how these maps are drawn

in order to be helped instead of unconsciously steered by them.

(Rovira-Esteva, Orero et Aixelá 2015: 160)

La légitimation de la discipline soulève la question de son statut: la traductologie est-elle une science? Holmes, en recherchant cette légitimité, a voulu placer ce qu'il a appelé translation studies parmi les disciplines empiriques. Son argumentation recèle cependant une contradiction. De plus, structurant la discipline, il a voulu tracer des chemins qui en permettaient l'exploration. Toutefois, l'opposition entre le pur et l'appliqué ne nous paraît plus rendre compte des rapports actuels entre théorie et applications.

\subsection{Traductologie (translation studies) et empirisme}

Holmes, en cherchant à justifier la présence du mot studies dans translation studies, plaçait la discipline hors du champ des sciences, opposée aux sciences humaines ou aux arts. Son argument, partant de l'allemand Wissenshaft, évoque singulièrement Schleiermacher:

There is, however, another term that is active in English in the naming of new disciplines. This is the word "studies». Indeed, for disciplines that within the old distinction of the universities tend to fall under the humanities or arts rather than the sciences as fields of learning, the word would seem almost as active in English as the word Wissenshaft in German. (Holmes 1972/2004: 183)

Pourtant, un peu plus loin, citant $\mathrm{Hempel}^{8}$, il place la toute nouvelle discipline au sein des disciplines dites empiriques, à l'aide d'une prolepse (" as no one I suppose would deny»), suivie d'un argumentum at populum («it is often pointed out»):

From this delineation it follows that translation studies is, as no one I suppose would deny, an empirical discipline. Such disciplines, it is often pointed out, have two major objectives, which Carl G. Hempel has phrased as «to describe particular phenomena in the world of our experience and to establish general principles by means of which they can be explained and predicted.» (Holmes 1972/2004: 184; nous soulignons)

Placer la traductologie (nous garderons ce terme pour éviter des confusions) à la fois hors des sciences, mais dans la filiation d'une pensée relevant du positivisme logique radical paraît cependant complexe à réconcilier. En effet, le positivisme logique, école de pensée du Cercle de Vienne, se caractérise par son empirisme strict et une volonté unificatrice de la science rejetant la réflexion métaphysique ou simplement spéculative: "Il n'y a de connaissance qu'extraite de l'expérience, c'està-dire de ce qui est immédiatement donné.» (Neurath, Carnap et Hahn. 1973: 309, cité et traduit par Malherbe 1976/2011: 44). Selon Schlick, le fondateur du Cercle, «la métaphysique disparaît [...] non parce que la tâche qu'elle s'est assignée dépasse les forces de notre raison, mais parce que la métaphysique n'existe pas!» (Malherbe 1976/2011 : 45). Prônant l'éducation pour tous (et surtout l'éducation scientifique), le Cercle de Vienne se place explicitement dans la filiation des Lumières, car «[d]ans 
l'esprit de la plupart de ses membres il y a une connexion évidente entre, d'un côté, la métaphysique, la théologie et la religion et, de l'autre le dogmatisme, l'autoritarisme et la réaction. Et il y en a une autre du même genre entre, d'un côté, la rationalité, la science, l'attitude antimétaphysique et la conception scientifique du monde et, de l'autre, le libéralisme, la démocratie et le progrès social et politique.» (Bouveresse 1997: 18).

Il n'est pas dans notre propos ici de positionner la traductologie dans un cadre épistémologique uniforme, qui serait, par exemple, une adhésion aux Lumières ou un rejet de celles-ci et des filiations qui s'ensuivent. Au contraire, nous voudrions souligner que cela n'est ni souhaitable, ni même possible: le caractère interdisciplinaire de la traductologie l'empêche fondamentalement. Et en cela nous partageons l'ouverture de Bensimon à la nécessité de préserver la diversité des approches.

Mais l'on voit bien le problème: Berman se situe dans une perspective héritée du romantisme allemand qui s'oppose aux Lumières. Est-il «hors traductologie» selon Holmes et son apparente adhésion à l'empirisme logique? Dans les faits, Holmes se voulait sûrement inclusif, mais épistémologiquement, sa justification paraît problématique.

En fait, chaque chercheur se rallie nécessairement à une filiation ou à une autre, ce qui contraint la traductologie à être un lieu de débat et non pas à être régie par une doxa de quelque obédience que ce soit. Ceci pour le meilleur et pour le pire, mais nous serions portée à envisager la chose de manière optimiste, et à souligner la richesse potentielle d'une telle situation. C'est d'ailleurs peut-être là que la traductologie pourrait se révéler la plus féconde par rapport aux disciplines avec laquelle elle entretient des relations, car devenant un terrain de mise à l'épreuve de cadres théoriques importés, elle sort de la position (relativement inconfortable) de l'interdiscipline pour devenir un lieu privilégié de mise à l'épreuve (terrain de réfutation), de synthèse et aussi d'exportation de nouveaux concepts et de nouvelles manières de regarder le monde. À titre d'exemple, citons le cas du rapport entre l'histoire et la traduction, dont l'apport dans les recherches en histoire est immense (voir par ex., Bastin 2004).

\subsection{La carte de Holmes: le pur et l'appliqué}

L'opposition entre une traductologie pure et une traductologie appliquée nous paraît une des sources des difficultés de transfert du résultat des recherches à la pratique de la traduction. Elle trouve, sinon son origine, tout au moins son ancrage, dans la structuration des translation studies de Holmes. Cette structuration, initialement présentée sous forme de texte (Holmes 1988), puis sous forme de graphe grâce à Toury (Toury 1995/2012: 4), est cependant un objet de référence quasi-universel en traductologie occidentale (Munday 2012: 16) (figure 1).

Pourtant, comme le souligne Chesterman (Chesterman 2009: 15), un certain nombre de critiques ont été formulées à l'égard de la carte de Holmes. Lui-même questionne les relations entre théorie et description (Chesterman 2004) et il observe qu'elle ne rend pas compte adéquatement des recherches axées sur le traducteur (Chesterman 2009: 13). On peut également citer les critiques suivantes: l'histoire n'est pas mentionnée (Pym 1998), les paramètres contextuels et pragmatiques ne sont pas suffisamment pris en compte (Lambert 1991), non plus que le caractère interdis- 
ciplinaire (Snell-Hornby 1991), Giles (2005) relève des problèmes soulevés par l'aspect descriptif.

Selon van Doorslaer, qui propose une série de graphes rassemblant bon nombre de concepts clés, personne ne s'est risqué à proposer une nouvelle carte pour remplacer celle de Holmes dès lors que Pym eût critiqué le simplisme des arborescences:

Yet is there any reason to suppose that the Holmes map is automatically suited to what we want to do in translation studies now? [...] No matter how pretty the maps, if a branch of scholarship fails to address socially important issues, it may deserve to disappear or to be relegated to academic museums... (Pym 1998: 2-3; cité dans Van Doorslaer 2007: 220).

De fait, la carte de Holmes nous paraît cristalliser le problème de fond de la traductologie, certes dans sa structure, mais de manière plus fondamentale, dans ce qu'elle sous-tend en termes de positionnement épistémologique:

1) Elle tente de synthétiser dans un graphe à deux dimensions un ensemble de concepts grâce à des liens tout/partie (Translation Studies => «Pure» / Applied) et générique/ spécifique (Descriptive => Product oriented / Process oriented / Function oriented).

Ce mode de représentation ${ }^{9}$ en arbre de Porphyre orienté est restrictif, car il ne permet pas de rendre compte des multiples paramètres pouvant être étudiés en traductologie. Nous pensons qu'il tend à bloquer la circulation des idées au sein même du domaine: si l'on s'intéresse au processus de traduction, est-il possible d'en envisager les répercussions au plan de la formation ou des outils? Une étude se penchant sur les processus cognitifs en pédagogie devrait-elle s'inscrire dans les études descriptives (pure/ process oriented) ou dans les recherches pédagogiques (applied/translator training)?

2) Elle oppose une traductologie "pure» et "appliquée», consacrant ainsi le caractère «noble» d'un certain type de recherche (surtout la «théorie», mais aussi les études descriptives).

Même si Holmes envisage les rapports entre le théorique, le descriptif et l'appliqué comme une dialectique (contrairement à Toury qui considère l'appliqué comme secondaire), la distinction posée à la racine même de la discipline nous semble problématique.

La carte de la traductologie Holmes reprise par Toury ${ }^{10}$ (Chesterman 2009: 14)

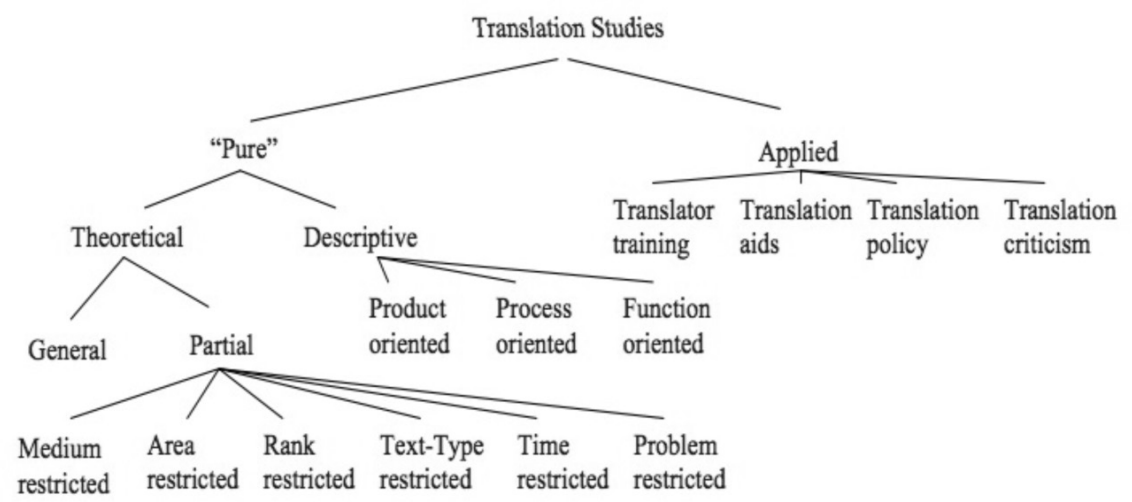


Selon Bensaude-Vincent, la division entre "pur» et "appliqué» ne serait redevable ni à Kant (qui oppose le «pur» à l'«empirique»), ni à Comte, qui

[...] utilise la distinction générale entre «spéculation» et «action», à laquelle il fait correspondre deux catégories de connaissance théorique et pratique, le couple de catégories sous-tend la fameuse formule du positivisme: «science d'où prévoyance, prévoyance d'où action». Tout en admettant qu'au temps de Bacon on ait progressé en concevant la connaissance «comme véritable base rationnelle de l'action de l'homme sur la nature», Comte déplore l'utilitarisme de son siècle qui réduit les sciences à cette fonction, ignorant leur destination essentielle qui est de connaître les lois des phénomènes. L'autorité des recherches théoriques par rapport aux besoins pratiques appelle, dit Comte, un médiateur entre science et arts, concrétisé dans une forme sociale nouvelle, l'ingénieur, «dont la destination spéciale est d'organiser les relations de la théorie et de la pratique». (Bensaude-Vincent 2008/2012: section 6)

Cette division prendrait sa source dans une « origine locale», celle de la chimie:

Les expressions "chimie pure» et "chimie appliquée» ont une origine assignable en précision. Le chimiste suédois Johan Gottschalk Wallerius, titulaire en 1750 d'une chaire de chimie, de médecine et de pharmacie à l'Université d'Uppsala, utilise ces expressions dans un traité de chimie publié en 1751. D’après Christoph Meinel, cette dichotomie correspond à une tentative de légitimation de la chimie sur la scène académique. Les notions de pur et d'appliqué permettent un renversement stratégique: la priorité chronologique des arts chimiques - teinturerie, verrerie, métallurgie, pharmacie, etc., - est effacée au profit d'une dépendance logique des arts à l'égard de la science. Le changement de statut participe, d'après Meinel, de la tradition allemande et scandinave caractéristique des Lumières qui mêle étroitement la chimie, le mercantilisme et le «kammeralisme». Les multiples traductions du traité de Wallerius ont permis une large diffusion des expressions "pur» et "appliqué» notamment en Allemagne et en Angleterre dans la deuxième moitié du XviII ${ }^{\mathrm{e}}$ siècle. Comme le souligne une étude de Robert Bud et Gerrylyn K. Roberts sur l'Angleterre victorienne, c'est dans un contexte de promotion de l'enseignement de la chimie que les expressions "pur» et "appliqué» font fortune. La catégorie «chimie pure» justifie la création de cours de chimie dans des formations généralistes autant que professionnelles. Car paradoxalement, c'est au nom de son utilité que la chimie est promue comme une science pure. (BensaudeVincent 2008/2012: section 8)

Bensaude-Vincent poursuit en expliquant la diffusion du rapport pur/appliqué notamment en France et le fait que, de nos jours, plus personne ne songe à s'y rattacher, sous l'influence des nouveaux modèles de recherche, issus des États-Unis dans le contexte de la Seconde Guerre mondiale et de la guerre froide et qui favorisent «le décloisonnement des disciplines, les partenariats entre recherche académique et industrielle, avec une forte sensibilité au marché et à la société» (Bensaude-Vincent 2008/2012: section 38).

On ne peut que regretter que la rupture entre le modèle linéaire du pur vers l'appliqué soit en fait soumis à des considérations mercantiles et même sociales: mais selon nous le problème vient surtout de la structuration des systèmes de recherche et de l'emploi rhétorique de ces distinctions ${ }^{11}$. Si nous suivons le raisonnement de Bensaude-Vincent, le couple théorie/pratique, bien mieux que pur/appliqué, manifeste la linéarité (ou mieux encore, l'itérativité) entre la réflexion du chercheur dédiée à la connaissance et au transfert de cette connaissance à des questions soulevées dans «le monde». 


\section{Théorie, pratique, et retour... vers le futur}

'Translation theory? Spare us...' That's the reaction to be expected from most practising translators.

(Chesterman et Wagner 2010)

Dans la tension théorique - descriptif, faut-il entendre par théorique ce qui est purement spéculatif? Quel est le sens historique de cette opposition? Qu'entend-on par théorie de nos jours? Est-il possible de faire une étude descriptive sans avoir un positionnement théorique, au sens du choix d'un cadre de réflexion assorti de prémisses lui servant de balises et permettant la constitution d'hypothèses, sous peine de dresser un catalogue de données n'ayant que l'induction pour seule issue? $\mathrm{Ne}$ peut-on pas, à partir du descriptif, tirer des conclusions remettant en cause les hypothèses? Quel est le rapport entre les applications et les théories? Enfin, question épineuse, les théories de la traduction ne seraient-elles pas plutôt des modèles et non des théories au sens moderne du terme?

\subsection{Théorie/spéculation et pratique: Aristote et sa filiation}

Le couple intellect spéculatiflintellect pratique remonte à Aristote (IV s. av. J.-C.), qui l'évoque dans l'Éthique à Nicomède (Aristote Iv s. av. J.-C./1959) et dans De l'âme (Aristote IV ${ }^{e}$ s. av. J.-C./1988/2010). On le retrouve dans la Somme de théologie de Saint Thomas d'Aquin (ca. 1225-1274), moine célèbre pour la dispute qui l'opposa à Avicenne et surtout pour sa synthèse de la tradition chrétienne et de la philosophie d'Aristote.

Ce qui nous intéresse davantage est la manière de représenter les connaissances. L'une des premières représentations connues est la Philosophiae partitio, de Gregor Reisch (ca. 1470-1525), moine de l'ordre des Chartreux ${ }^{12}$, présentée dans sa Margarita philosophica (La Perle philosophique; Reisch 1496/1503 or $1504^{13}$; figure 2). Souvent présentée comme la première encyclopédie, la première version de 1503 précède cependant la naissance du terme encyclopedia d'une trentaine d'années (Cunningham et Kusukawa 2010: ix) : il s'agit plutôt d'un précis, d'un condensé (epitome) à l'intention des jeunes gens.

Elle se caractérise par une division de la philosophie - c'est-à-dire de la science, pour le monde médiéval - mettant, d'un côté, le théorique et le spéculatif, de l'autre, la pratique, dans une perspective conciliant, curieusement, les héritages aristotélicien et, par l'intermédiaire de Saint Augustin, platonicien (Cunningham et Kusukawa 2010 : xiii). Concernant en fait l'organisation de l'université médiévale (et c'est en cela qu'elle se distingue de la réédition des textes d'Aristote [Cunningham et Kusukawa 2010 : xi]), elle vise à classer les domaines de connaissance, et non à établir un rapport entre une réflexion théorique et ses applications. La Perle philosophique s'inscrit dans le cadre de la querelle des universaux ${ }^{14}$, où s'affrontent des théories héritées d'Aristote ou de Platon ${ }^{15}$. Ne cherchant pas à trancher le débat, les divisions de la Philosophiae partitio sont en fait un «montage» des catégories de la connaissance médiévale visant à résumer cette dernière (Cunningham et Kusukawa 2010: xxxv; nous renvoyons le lecteur à cette référence pour plus de détails).

La séparation de la philosophie théorique, ou spéculative, et de la philosophie pratique reprend les divisions de Boèce ${ }^{16}$ (ca. 470-524) dans De la Trinité. La philosophie 
FIGURE 2

Les parties de la philosophie, selon Gregor Reisch (1496/1503 ou 1504)

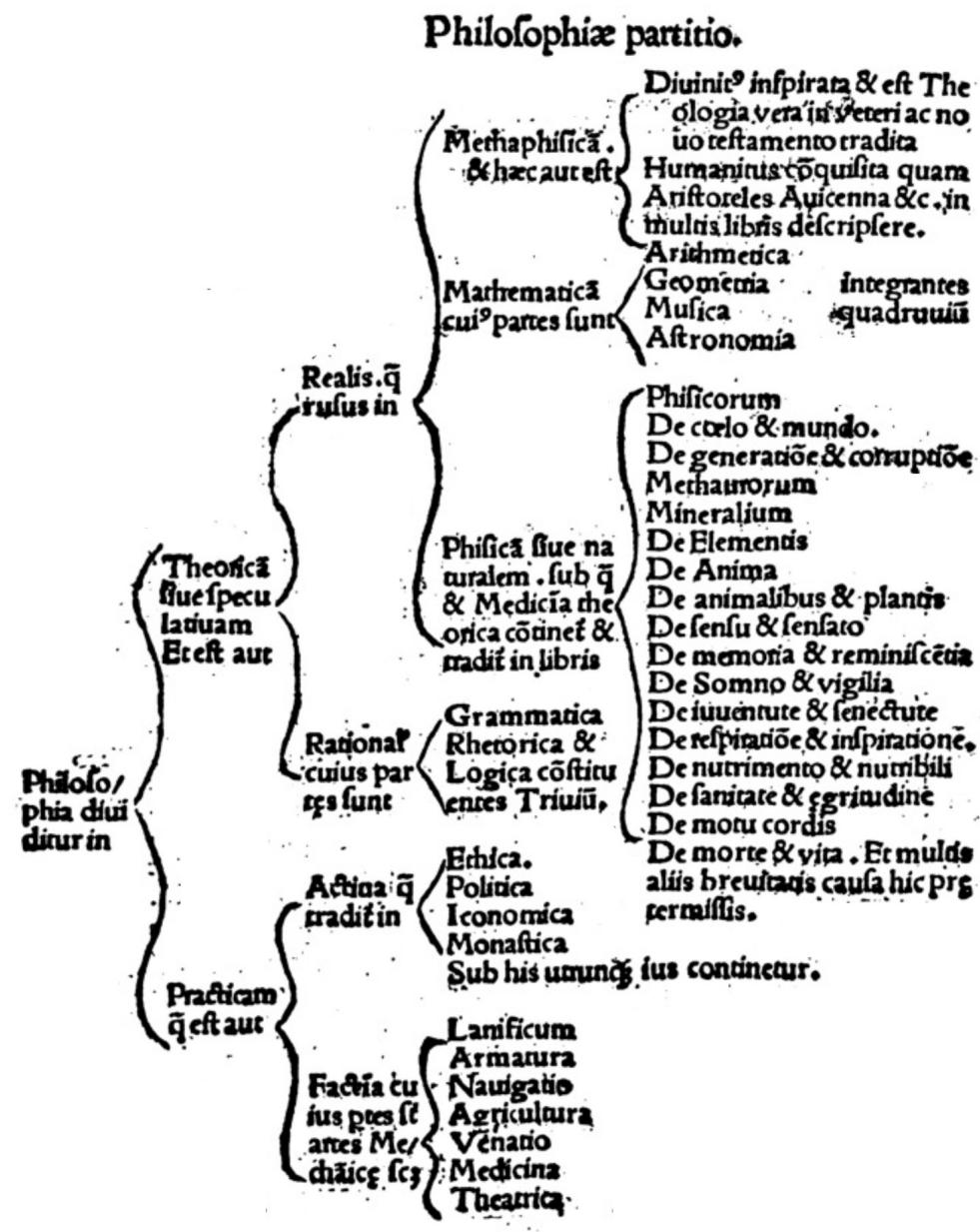

théorique est divisée en philosophie réelle (qui s'occupe des choses [res] : métaphysique, mathématique, physique [qui inclut la médecine, dans son aspect théorique]) et en philosophie rationnelle (grammaire, rhétorique, logique). La philosophie pratique, dans son versant actif, comprend l'éthique, la politique, l'économie, la monastique ainsi que le droit canon et la loi civile. Dans son versant productif, elle comprend sept arts mécaniques: le tissage, la fabrication des armures, la navigation, l'agriculture, la chasse, la médecine (qui apparaît une deuxième fois, ici dans son aspect pratique) et le théâtre.

Bien entendu, la nature des disciplines est assez loin des divisions de la carte de Holmes, mais on y retrouve une logique semblable, notamment la disjonction entre ce qui relèverait de la réflexion et ce qui relèverait de l'action. Nous ne sommes pas ici dans une logique de relation entre la recherche et ses applications. La séparation de la médecine en ces deux dimensions est particulièrement illustrative, parce que 
l'on peut facilement comprendre que ces catégories ne s'appliquent plus de nos jours. Ensuite, ce type de représentation correspond à une catégorisation arborescente en fonction de différents paramètres: c'est un arbre de Porphyre.

Reporté sur la traductologie, ce mode de représentation contient une telle rupture entre le monde universitaire et la pratique professionnelle qu'il ne faut pas s'étonner des conséquences: la séparation entre une réflexion et une action difficiles à réconcilier est la source, à notre avis, du malentendu. Par conséquent, nous posons que le graphe arborescent de Holmes est devenu inadéquat pour rendre compte des dimensions variées de la traductologie. Il constitue une métaphore visuelle qui tend à figer la réflexion, car il ne permet pas de conceptualiser aisément les relations entre ses constituants.

Mais avant de proposer un autre mode de représentation, il nous faut clarifier la nature de la théorie.

\subsection{La théorie comme outil de représentation et d'inférence}

Retracer l'histoire du concept de théorie depuis l'antiquité dépasse très largement le cadre du présent travail. Nous ferons un saut dans l'histoire des sciences pour nous intéresser au concept de théorie aujourd'hui. Pour mieux ouvrir l'avenir, posons qu'il ne saurait être question de confiner la théorie traductologique à son versant strictement spéculatif, et qu'il s'agit de dépasser les oppositions entre Lumières et romantisme, ou entre positivisme et postmodernisme: de fait, ces oppositions sont obsolètes, car nous en sommes (enfin!) à une conciliation raisonnée de positions extrêmes, comme l'expose Vorms (2009).

Vorms adopte comme prémisse, dans ses présupposés de recherche (auxquels nous adhérons), qu'une théorie est un «outil de représentation et d'inférence» qui se distingue d'un «simple compte-rendu d'observations par le fait qu'elle exprime des hypothèses générales à propos des phénomènes qu'elle décrit.» (Vorms 2009: 1):

Une théorie est à la fois un outil de représentation et un outil d'inférence ou de calcull ${ }^{17}$. Ces deux ingrédients sont indispensables à la définition d'une théorie scientifique: si l'une de ces deux fonctions n'est pas remplie, on refuse généralement de parler de théorie scientifique. (Vorms 2009: 1)

Cette définition contient en elle-même le rejet de l'induction pure, c'est-à-dire le fait que la simple collection de données ne saurait mener à une théorie (ce qui n'empêche pas qu'il y ait une phase inductive de la recherche, laquelle sert pour obtenir les données préliminaires à l'établissement d'une question de recherche). La fonction «outil de représentation» est essentielle: Vorms souligne qu'une théorie est bien plus qu'un simple instrument de calcul (au sens restreint), comme un ordinateur qui pourrait formuler des résultats cependant justes. Son hypothèse de travail est que "c'est en vertu de la manière dont elle représente les phénomènes qu'une théorie permet de tirer des inférences» (Vorms 2009: 3; souligné par l'auteure). Une théorie est ainsi «à la fois un discours et un instrument», ces deux aspects dépendant l'un de l'autre. Le point qui nous paraît absolument fondamental, c'est l'aspect représentation: «c'est en fournissant une image «newtonienne» du monde [...] que la mécanique permet de faire des calculs qui conduisent à l'explication et à la prédiction du mouvement des corps» (Vorms 2009: 4). Nous voici revenus à «l'expérience de mondes» autorisée par la manière dont les théories sont construites. 
Vorms mentionne, sans entrer dans les détails, les débats suscités par la question de l'explication utilisée par les positivistes: il ne faut pas entendre par explication «l'élucidation des causes premières des phénomènes observables», qui sont des "purs objets de spéculation» métaphysique: autrement dit, on peut poser la question du comment, mais pas celle du pourquoi. Dans la perspective positiviste, «la théorie est bien un outil de représentation et d'inférence, mais les explications qu'elle fournit sont les stricts analogues des prédictions; elles ont la même forme logique, une forme hypothético-déductive.» (Vorms 2009: 4)

Surtout, Vorms ouvre une porte qui permet de sortir de l'apparente impasse créée par la prise de conscience que les théories scientifiques sont des constructions de l'esprit humain, largement exploitée par les courants postmodernes pour critiquer le positivisme.

\section{Elle rappelle que la tradition positiviste}

[...] se caractérise par le refus de prendre en considération la dimension psychologique de l'utilisation des théories par les agents. [...] En conséquence, les positivistes logiques ainsi que leurs héritiers étudient la double fonction des théories (représentation et inférentielle) d'un point de vue logique et non pas d'un point de vue cognitif: ils définissent le contenu des théories comme tout ce que l'on peut en déduire, en principe, en vertu de règles objectives, mais ne prêtent pas attention aux processus inférentiels que les agents mettent effectivement en œuvre. (Vorms 2009: 4)

Sa thèse prend une position, sur ce point, diamétralement opposée, et c'est ce parti pris qui permet de résoudre enfin l'impasse découlant du conflit, insoluble, entre positivistes et postmodernes:

Je propose d'examiner la double fonction des théories en adoptant la perspective de leurs utilisateurs. Par «utilisateurs d'une théorie», j’entends désigner aussi bien les experts qui l'élaborent, la développent et la convoquent pour prédire et expliquer les phénomènes, que les étudiants qui l'apprennent. Adopter cette perspective revient à considérer les théories comme les productions d'esprits humains, et à étudier leur fonctionnement comme outils de représentation et d'inférence sous l'angle de leur utilisation, en pratique, par des agents aux capacités cognitives limitées. Autrement dit, je pars du principe qu'il n'y a pas de théorie sans théoricien ou, plus largement, sans des agents qui l'utilisent pour représenter les phénomènes et pour tirer des inférences à leur propos. (Vorms 2009: 5; nous soulignons)

Cette position permet de rendre compte de l'activité scientifique pour ce qu'elle est, à savoir une activité humaine mobilisant la cognition (et donc en dépendant), se voulant tendue vers l'objectivité, mais sujette aux aléas de la subjectivité; elle permet d'intégrer le réel dont l'appréhension ne peut être que partielle; enfin, elle permet de rendre compte du cheminement de la construction des savoirs (dans la version plus subjective) ou de l'accumulation des connaissances (dans la version plus objective).

\subsection{Quelques propositions métalangagières}

Il est impossible d'imaginer qu'une seule théorie rende compte de tous les aspects de la traductologie. Le positionnement théorique sera en fait défini par l'objet à l'étude, la question posée et les prémisses adoptées pour aborder la question. Les prémisses ne sont pas démontrables, mais les hypothèses que l'on peut formuler à partir d'un cadre théorique doivent l'être, que ce soit par une recherche de type qualitatif ou quantitatif. 
Ainsi nous nous sommes expliquée sur nos choix de cadres théoriques pour aborder une traductologie cognitive ${ }^{18}$ (Vandaele 2000; mais surtout Vandaele 2007), que nous rattachons à une filiation de pensée remontant à Spinoza plutôt qu'à Descartes: au lieu de postuler le corps-machine, nous adoptons, comme prémisses, l'optique du caractère substantiel de la nature et avec elle l'unicité du corps et de l'esprit. De là découlent un certain nombre de conséquences, notamment l'exclusion de cadres dualistes cartésiens et l'adoption d'un cadre cognitif expérientialiste appliqué au langage (Lakoff et Johnson 1985/2003), ce qui nous permet d'envisager l'étude des modes de conceptualisation scientifique à partir de corpus de textes scientifiques.

Le plus souvent, en traductologie, la réflexion sur la pratique traduisante est qualifiée de théorie. Nous sommes convaincue que parler d'une métaréflexion menant à des modèles serait plus éclairant - surtout pour les jeunes chercheurs. Ainsi, l'équivalence dynamique de Nida, l'incitation à l'étrangéisation de Venuti et de Berman, le fonctionnalisme de Nord nous paraissent être des modèles qui peuvent être utilisés soit par le praticien pour établir ses choix traductionnels, soit par le chercheur pour questionner la pratique d'un traducteur dont il analyse le travail.

Toutefois, cette métaréflexion ne se fait pas dans un vide théorique. Nida se place dans un cadre de syntaxe générative chomskyenne, Berman, nous l'avons vu, situe sa réflexion dans la filiation de Schleiermacher et du romantisme allemand, de même que Venuti tandis que Nord se réfère à la théorie de l'agir. Ceci permet de comprendre les débats au sein de la discipline. On pourrait se poser la question de savoir s'il faut se voir comme un héritier des Romantiques pour, dans la pratique de la traduction, adopter une approche étrangéisante. La convergence possible des aboutissements de cadres théoriques distincts est en fait une question passionnante que nous ne pourrons aborder ici.

\section{Carte, graphe et théorie}

\subsection{Multidimensionnalité du concept de traductologie}

Cette réflexion mène à proposer une représentation de la traductologie:

- qui permette d'aborder l'objet de toutes les manières possibles;

- qui permette de concevoir les itérations entre théorie(s), entendues comme outil de représentation et d'inférence, et pratique, à partir de laquelle la phase inductive de la recherche opère et où les hypothèses sont mises à l'épreuve;

- qui ouvre la possibilité d'interactions avec d'autres disciplines, ainsi qu'avec les cadres théoriques et les outils méthodologiques pertinents.

Gardons la métaphore de la carte, mais rappelons qu'il s'agit d'un graphe, non orienté. Nous proposons de le construire à partir du «centre actif», l'acte de traduction. Le traducteur est l'agent essentiel à cet acte - mais celui-ci pourrait être aussi mené de manière totalement autonome par un système informatisé. Partir de l'acte permet donc déjà d'inclure dans la réflexion la diversité des agents traduisants, qu'ils soient humains ou non - et que cela nous plaise ou non: c'est dans l'acte de réflexion que les positions en faveur ou contre pourront être correctement argumentées, et non dans l'effacement a priori de ce qui pourrait déranger.

L'acte de traduction est par essence un supraconcept multidimensionnel. Les différentes dimensions (fatalement représentées dans un plan en raison de la 
FIGURE 3

Carte de la traductologie au $\mathbf{X X I}^{\mathrm{e}}$ siècle

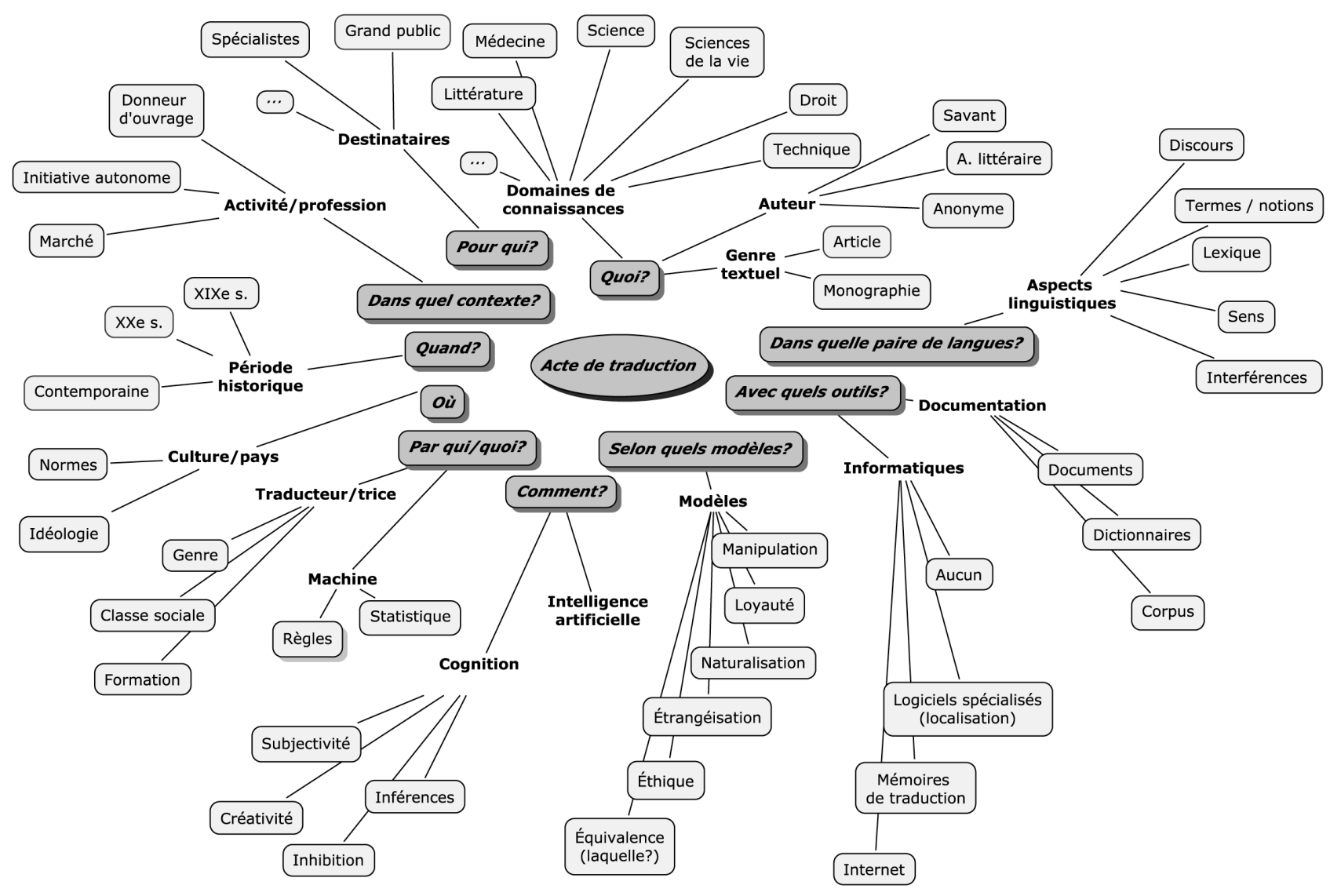

* Une version moins étoffée a été présentée au congrès de la CIUTI à Beijing, 21-22 mai 20119. 
contrainte de l'article) de ce supraconcept sont représentées par des questions insérées dans des boîtes gris foncé associées à des valeurs (en gras, boîtes sans contour). Celles-ci peuvent se décliner à l'infini par des concepts représentés dans les cases grises. Nous insistons sur le fait que cette carte est perfectible et évolutive: ceci est symbolisé par les cases contenant trois points (qui n'ont été indiquées que deux fois par souci de ne pas la surcharger, mais qui auraient dû apparaître pour chaque dimension). Les différents concepts construits ou explorés depuis les débuts de la traductologie s'insèrent dans la carte à sa périphérie.

La carte peut être utilisée de différentes manières:

- pour situer une pratique de traduction et déterminer les paramètres d'une métaréflexion;

- pour délimiter une question de recherche et construire la méthodologie, grâce à l'identification des variables dépendantes et indépendantes;

- pour délimiter des sous-domaines de la traductologie;

- pour délimiter des éléments d'un programme d'enseignement.

Nous insistons sur le côté évolutif de la carte, et sur le fait qu'elle peut - et doit être constamment mise à jour et complétée. Brems, Meylaerts et van Doorslaer (2012: 3) soulignent l'explosion des nouveaux domaines de recherche en lien avec les différentes pratiques professionnelles: apparition de nouveaux outils, de nouvelles pratiques, de nouveaux champs de traduction, comme la localisation, etc. Ainsi l'appel de Pym quant à la nécessité de rendre compte de l'influence de l'évolution de la profession sur la traductologie est-il pris en compte.

\subsection{Retour à la théorie}

Ce que l'on nomme habituellement théorie en traductologie est souvent un ensemble de principes fondant un modèle de la pratique de la traduction, ce modèle s'inscrivant, lui, dans un cadre théorique. C'est ce que Froeliger met en évidence lorsqu'il analyse les positions respectives de Meschonnic, Nida, Nord et Taber (Froeliger 2010).

Dans le modèle de carte que nous proposons, la notion contemporaine de théorie s'intègre parfaitement (bien qu'elle ne soit pas représentée graphiquement). L'objet d'étude et les variables à prendre en compte détermineront les théories possibles. Le chercheur déterminera à laquelle il adhère en fonction de son accord avec ses prémisses. Ainsi, dans notre cas, avons-nous souscrit aux cadres théoriques complémentaires de Lakoff, Fauconnier et Talmy, relevant de la sémantique cognitive, pour analyser la conceptualisation métaphorique dans les sciences biomédicales (Vandaele 2007). Une analyse des relations sémantiques pourrait relever de la Théorie Sens-Texte (TST) de Mel'Cuk, comme l'ont fait d'abord Dancette, puis L'Homme pour élaborer leurs dictionnaires spécialisés. La mise à l'épreuve de la théorie dans le cadre de l'objet étudié peut mener à une réfutation des prémisses initiales: nous avons ainsi constaté que les prémisses de la TST, qui déclare ne s'intéresser qu'aux relations entre unités lexicales, ne permettaient pas de prendre en compte le terme entendu comme unité de langue renvoyant à une notion (Vandaele et Raffo 2008). De fait, une des conditions posées par la TST est qu'elle exclut l'étude du rapport au monde. Pour cela, il faudra avoir recours à une sémantique référentielle telle que celle qui est proposée par Kleiber (voir Vandaele, Gendron-Pontbriand et Olivier-Bonfils, 2013). 


\section{Quelques propositions pour une traductologie des domaines de spécialité}

La complexité, autant que la valeur, de la traduction dans les domaines de spécialité est parfois sous-estimée de manière étonnante. Ainsi Dollerup ramène-t-il la traduction pharmaceutique à la traduction d'une liste de composés; et quand la compréhension fait défaut, pourquoi ne pas se contenter d'un mot-à-mot et laisser au lecteur le soin de «casser le code»?

In the present-day world, we do meet with word-for-word in professional translation in some specific instances. One such field is in the pharmaceutical industry. Why? It is done in order to make sure that every component in the product is listed. Another area is one well-known to experienced translators but not mentioned in public: you are translating a specialised text and all of sudden you hit upon words, strings of words, and passages which you do not comprehend. What do you do? The only option is to translate them word for word. In my experience, it works in at least $80 \%$ of the cases. Why? Because the clients and the audience have the background knowledge that enables them to crack the code. (Dollerup 2005)

Avec une telle affirmation, autant laisser les systèmes de traduction automatique se débrouiller à moindre coût, le lecteur séparera le bon grain de l'ivraie! Nous nous opposons vigoureusement à une telle approche de la traduction spécialisée - et ce avec d'autant plus d'affirmation que l'exemple pris par Dollerup tombe, précisément, dans notre champ d'expertise. Non seulement la traduction dans le domaine pharmaceutique est très loin de se limiter à une traduction de liste de composés, mais l'option du mot-à-mot et du renoncement à la compréhension est autant inconcevable qu'irresponsable, il est dans la droite ligne de préjugés négatifs à l'égard de la traduction spécialisée. Or, les enjeux sont majeurs, puisqu'il s'agit de la santé20. Le même raisonnement s'applique dans tous les autres domaines.

Voici donc quelques pistes, influencées, bien sûr, par notre propre expérience de la pratique, de l'enseignement et de la recherche. Il est entendu que dans chacune des catégories envisagées ci-dessous plusieurs chercheurs ont déjà réalisé un ample travail, mais en raison de l'espace, nous nous bornerons à ne présenter que nos propres réflexions.

\subsection{Les agents de la traduction}

On sait relativement peu de choses sur les pratiques professionnelles effectives dans différents contextes d'exercice. Quels sont les rapports entretenus avec les donneurs d'ouvrages, qu'il s'agisse de clients ou d'employeurs? Qui devient gestionnaire de services linguistiques et quelle influence exerce-t-il sur la pratique? Comment cela se traduit-il sur le plan des résultats et de la qualité? Sur celui du statut de la profession? Quels sont les débouchés autres que la traduction proprement dite? Les traducteurs se heurtent-ils à des questions d'éthique et quelle est leur marge de manœuvre? Les associations et les ordres professionnels sont généralement le lieu où l'on mesure l'évolution des conditions de travail, mais de manière ponctuelle et nécessairement partielle. On sait, généralement, que les traducteurs ont encore des difficultés à s'affirmer devant le client. Bien que des progrès aient été réalisés, l'atomisation de la pratique en travail autonome est un facteur d'affaiblissement. Des 
études sociologiques et/ou ethnographiques seraient donc précieuses pour informer les programmes d'enseignement et mieux préparer les futurs professionnels à prendre la place qui leur revient. Ces questions sont en fait très générales et s'appliquent à tous les types d'exercice de la traduction.

\subsection{Les genres textuels spécialisés}

Depuis quelques années, des travaux fructueux portant sur les genres textuels ont été entrepris (voir par exemple les travaux du groupe GENTT en Espagne (Montalt Resurrecció et García Izquierdo 2002, Montalt Resurrecció, Ezpeleta Piorno et García Izquierdo 2008). Les paramètres du genre et du type textuels constituent de fait des éléments clés des choix traductionnels. Beaucoup reste à faire cependant pour mieux les comprendre et offrir aux étudiants et aux praticiens des repères effectifs dans les différents domaines spécialisés - surtout dans une perspective d'analyse et de choix raisonnés et non dans une perspective prescriptive et normative. Pour le moment, les compétences en rapport avec les genres textuels s'acquièrent surtout par l'expérience, une fois un certain défrichage réalisé dans le cadre des formations.

\subsection{De la terminologie au discours spécialisé}

Rogers (2015) souligne que la majeure partie des recherches dans les domaines de spécialité ont été réalisées en terminologie, mais que la terminologie n'est pas tout. De fait, le travail réalisé dans différents contextes est véritablement imposant: pensons, pour le Canada, à Termium, pour le Québec, au Grand dictionnaire terminologique, sans compter tout ce qui s'est fait au sein des entreprises. Et n'oublions pas les entreprises de normalisation menées au sein des domaines de spécialité euxmêmes, comme le thésaurus constitué par les Medical Subject Headings (Vandaele 2013) ou les nomenclatures d'anatomie (Vandaele et Gingras-Harvey 2013). Cependant, ce qui a motivé un certain nombre de recherches était plutôt un intérêt pour la représentation des connaissances, motivé par les applications en intelligence artificielle, laissant souvent tomber, au passage, les composantes liées à la traduction humaine: compréhension fine de la mise en équivalence des dénominations, commensurabilité des systèmes conceptuels [voir Lakoff 1987/1990], problématique de la phraséologie [à laquelle notre intérêt pour les modes de conceptualisation est relié, voir Vandaele 2000], modalités cognitives du traitement terminologique chez l'apprenant ou le praticien.

Surtout, la composante discours n'a à notre sens pas été assez envisagée, la manière dont s'expriment les notions en discours selon les contextes n'étant pas prise en charge par les théories terminologiques, mais bien mieux par les approches de sémantique référentielle comme celle de Georges Kleiber (Vandaele, GendronPontbriand et Olivier-Bonfils 2013). Celles-ci permettent de mettre en évidence les différences de représentation subtiles mais complexes de représentations notionnelles et référentielles entre les langues et les cultures - réfutant ainsi le caractère mécanique et aculturel postulé par Ortega y Gasset ou Berman. 


\subsection{Les processus cognitifs et les enjeux de la formation}

Bon nombre de travaux ont permis d'ouvrir «la boîte noire» (en fait un concept behavioriste dépassé...) et de mieux comprendre comment le cerveau du traducteur fonctionne: analyse des mouvements oculaires, enregistrement de l'usage du clavier, protocoles de verbalisation, interactions avec les neurosciences pour recourir à l'imagerie (voir par ex. Shreve et Angelone 2010). Nous pensons qu'il faudrait se pencher sur les conséquences, sur le plan cognitif, de l'usage d'Internet et des outils de TAO, ainsi que des changements dans le monde professionnel. Nous avons ainsi vu apparaître, ces dernières années, des comportements en classe qui ont des conséquences radicales sur la formation et sur les stratégies d'enseignement: une diminution drastique de l'utilisation des dictionnaires, même électroniques; un recul de l'utilisation raisonnée des monographies et des articles spécialisés au profit de stratégies ponctuelles peu élaborées à l'aide de moteurs de recherche (le «tout-Google»); le tronçonnage du discours pour trouver des solutions toutes faites dans des concordanciers bilingues, assemblées ensuite à la manière de pièces d'un puzzle, au détriment de l'acquisition d'une expertise structurée d'un domaine de spécialité. Pourtant, les résultats préliminaires issus d'une petite étude-pilote que nous avons menée en classe montrent que les étudiants qui adoptent des stratégies paraissant plus coûteuses en temps au début de l'apprentissage sont, en fin de session, plus performants, en temps et en qualité, que ceux qui conservent des stratégies ponctuelles, et qu'ils sont plus à même de réviser des textes - tâche qui va devenir essentielle avec les systèmes de prétraduction automatique. Pour assurer aux traducteurs une position d'expert dans leur domaine en situation de maîtrise des outils informatiques (et non en victimes des systèmes automatisés), repenser la pédagogie dans une perspective cognitive est une urgence.

\subsection{Les perspectives historiques}

Nous terminerons par un domaine auquel nous nous intéressons depuis quelques années, celui de l'histoire des sciences. C'est un domaine encore très jeune en traductologie des domaines de spécialité, à la jonction des langues et de l'histoire des sciences, des techniques et des cultures (voir par ex. Duris 2008; Chevrel, D’hulst, et coll. 2012; Vandaele et Gendron-Pontbriand 2014). Sans aucun doute, le résultat des études déjà réalisées constitue une réfutation absolue de l'hypothèse d'aculturalité de l'écrit scientifique. Nous avons pu mettre en évidence, également, qu'une traduction de thésaurus comme celui des Medical Subject Headings pouvait avoir comme conséquence l'effacement d'un pan de l'histoire des sciences d'une culture (Vandaele 2013).

Soulignons que l'étanchéité hypothétique de la frontière entre écrit scientifique et écrit littéraire ou philosophique est également mise à mal (nous pensons notamment au cas de Darwin et de ses contemporains, mais aussi à tout un pan de l'écriture scientifique destinée à un lectorat averti, comme les essais écrits par de grands chercheurs comme Gerald Edelman, Jean-Pierre Changeux, Jean-Didier Vincent, François Jacob, etc.). 


\section{Conclusion}

Les particularités des traductions littéraires et non littéraires ne doivent pas justifier la prééminence de l'une par rapport à l'autre. Contrairement à Berman, Newmark en souligne plutôt les points de convergence:

They differ essentially through intention (literary texts belong to the world of imaginationwhereas non-literary ones belong to the world of facts) and through the fact literary texts are about persons while non-literary ones are about objects. Nevertheless, both texts are concerned with the fundamental truths of translation: factual, aesthetic, allegorical truth, logical and linguistic truth. (Newmark 2004:8)

Nous avons évoqué, au début de notre article, Berman, pour qui la littérature permet «l'expérience d'un monde». Le collectif dirigé par Duris (2008), qui explore l'histoire de la traduction scientifique et technique, met clairement en évidence que, peu importe le lieu, l'époque ou la discipline, la «traduction de textes scientifiques et une entreprise intellectuelle complexe, qui nécessite une profonde réflexion sur le monde scientifique».

Pour le courant expérientialiste, auquel nous nous rattachons, l'expérience du monde sous-tend le langage, et ce, dans la vie quotidienne même. De manière intéressante, Lakoff (1987/1990 : 322-323), auteur dont nous nous sommes abondamment inspirée pour notre travail sur la conceptualisation métaphorique, considère que la traduisibilité est l'une des conditions de commensurabilité de deux systèmes conceptuels. Mais c'est peut-être l'inverse! Et si les systèmes ne sont pas d'emblée commensurables, ils peuvent évoluer, s'influencer et le devenir: la traduction est aussi un instrument de commensurabilité des cultures.

Nous ajouterons que l'affirmation de la traductologie, en tant que discipline, et de la traduction, en tant que profession, demande encore des efforts: malgré un corpus maintenant imposant de monographies et de revues savantes, des programmes universitaires formant des doctorants et des associations actives - et même un ordre professionnel au Québec (l'OTTIAQ) - la traductologie - sinon la traduction - est encore confondue par certains avec la linguistique appliquée, notamment par les organismes subventionnaires et certaines entités internationales. Dans cette époque qui a la manie de tout classer, cela a des conséquences concrètes: par exemple, les revues de traductologie sont mélangées à des revues de linguistique dans la liste de l'ERIH (European Reference Index for the Humanities), ce qui crée un biais dans le classement (ce qui favorise clairement certaines revues de linguistique fonctionnant presque comme des revues médicales, puisque plus à même de suivre les normes rigides des index de citation). Par ricochet, cela a des conséquences pour l'établissement des financements et donc la survie de la discipline.

Malgré les obstacles à franchir, au vu de la richesse des territoires qui restent à explorer, il y a lieu de se réjouir et de «se retrousser les manches». S’il est un danger qui menace la traductologie, ce n'est pas, selon nous, son ouverture à l'interdisciplinarité, ni la multiplicité des sujets potentiels de recherche. Ce serait plutôt le risque de sclérose qui découlerait d'une conception des savoirs reposant sur des modèles antiques et dépassés. 


\section{REMERCIEMENTS}

Nous remercions Eve-Marie Gendron-Pontbriand pour sa traduction du résumé en anglais et Delphine Glerrer-Bonfils pour sa relecture attentive, ainsi que toute l'équipe qui a organisé le colloque en l'honneur du $60^{\mathrm{e}}$ anniversaire de Meta et mené à bien la publication du présent numéro, et en premier lieu, mon collègue et successeur à la direction de la revue, Georges Bastin.

\section{NOTES}

1. Scarpa regroupe l'ensemble de la traduction pragmatique sous le vocable de traduction spécialisée (Scarpa 2010). Cela inclut non seulement la traduction dans les domaines spécialisés - ce dont nous nous occupons - mais aussi la traduction audiovisuelle, la localisation, le sous-titrage, etc. Comme elle, nous jugeons que le vocable traduction générale est sujet à caution: en dehors de sa pertinence opératoire dans les programmes d'enseignement (un cours de traduction générale devrait permettre l'acquisition des principes de base de l'opération traduisante), il ne correspond à rien de précis, puisque, dans les faits, un traducteur dit général passera en fait d'une spécialité à l'autre même si le caractère spécialisé peut rester superficiel. Il s'agit de la même problématique que celle de la langue générale, souvent exemplifiée, dans des corpus, par des textes tirés de journaux: mais dans les faits, un journal contient des articles (un genre textuel à part entière) traitant de différents sujets, plus ou moins en profondeur, de la crise économique au sida en passant par la politique.

2. Références à l'appui, Brian Harris expose, dans un blogue mis en ligne en décembre 2009, sa version de l'origine des termes utilisés pour nommer la discipline: lui-même, en 1973, proposa translatology en 1973, ainsi que traductologie, ce dernier terme étant également employé par Jean-René Ladmiral. Alors que traductologie eut du succès (et fut traduit littéralement dans de nombreuses langues), c'est translation studies, proposé par Holmes, qui éclipsa son rival en anglais. Harris mentionne que translatology recueille encore $5 \%$ des suffrages, et que Peter Newmark, peu de temps avant sa mort, changea d'idée pour préférer translatology à translation studies. Bien que certains pensent que translatology et translation studies réfèrent à des contenus disciplinaires légèrement différents, nous n'avons pas ce problème en français: la traductologie englobera tout ce qui concerne la traduction, et généralement elle inclut aussi l'interprétation. Et tout comme translatologist en anglais, Harris souligne que le dérivé nominal traductologue séduit par sa fonctionnalité - ce qui est bien la moindre des choses! La querelle porte en fait sur le caractère hybride grécolatin de traductology (voir Harris 2009 et Holmes 1972/2004). On retrouve la même querelle ailleurs, notamment en médecine, dont la doxa traditionnelle tend à rejeter l'hybridation. En pratique, de nombreux hybrides grécolatins sont bien en usage, et pas seulement en médecine! (Voir Walter 2006).

À noter que Holmes (1972/2004) attribue à Roger Goffin (1971) la création de translatology et de traductologie. Goffin commente en fait traductiologie et non traductologie (Goffin 1971: 59). Pour mémoire, rappelons que la version publiée dans l'anthologie de Venuti est commentée, par Holmes lui-même, comme étant une version prépublication d'un texte rédigé en août 1972. Le fait que le texte de Holmes ait été publié en 1988 chez Rodopi rend complexe l'établissement des antériorités. Cependant, le terme translation studies apparaît dès 1971 dans un collectif dirigé par Holmes (1971), comme titre de la collection dont il est le premier volume.

Harris, Brian (2009): Translation Studies or Translatology? In: Brian Harris (2009- ): Unprofessional translation. Blogue consulté le 12 juin 2015, <http://unprofessionaltranslation. blogspot.ca/2009/12/translation-studies-or-translatology.html>.

3. Il s'agit de l'emplacement sur la version Kindle.

4. "Dem Gebiete der Kunst und der Wissenschaft eignet die Schrift, durch welche allein ihre Werke beharrlich werden; und wissenschaftliche oder künstlerische Erzeugnisse von Mund zu Mund zu dolmetschen, ware eben so unnütz, als es unmöglich zu sein scheint. Den Geschäften dagegen ist die Schrift nur mechanisches Mittel; das mündliche Verhandeln ist darin das ursprüngliche, und jede schriftliche Dolmetschung ist eigentlich nur als Aufzeichnung einer mündlichen anzusehen.» (Schleiermacher 1813/1985/1999: 34; nous soulignons)

"Au domaine de l'art et de la science convient l'écrit, par lequel seul ses œuvres perdurent; la transposition orale d'une production scientifique ou artistique serait aussi vaine qu'impossible. Pour les affaires, en revanche, l'écriture n'est qu'un moyen mécanique; le commerce oral est ici primordial, et chaque transposition écrite ne doit être considérée, à proprement parler, que comme l'enregistrement d'une transposition orale.» (Schleiermacher 1813/1985/1999: 35, traduit par Berman; nous soulignons) 
5. Bastin (2005: 122-123) rapporte que, selon García Yebra lui-même, Berman se serait inspiré de sa traduction pour traduire Schleiermacher.

6. «Una lengua es un sistema de signos verbales merced al cual los individuos pueden entenderse sin previo acuerdo, al paso que una terminología sólo es inteligible si previamente el que escribe o habla y el que lee o escucha se han puesto individualmente de acuerdo sobre el significado de los signos. Por eso la llamo pseudolengua y digo que el hombre de ciencia tiene que comenzar por traducir su propio pensamiento a ella. Es un volapuk, un esperanto establecido por convención deliberada entre los que cultivan esa disciplina. De aquí que sea más fácil traducir estos libros de una lengua a otra. En realidad, los de todos los países están ya escritos casi íntegramente en la misma. Tan es así que estos libros parecen herméticos, ininteligibles o por lo menos muy difíciles de entender a los hombres que hablan la lengua auténtica en que aparentemente están escritos. » (Ortega Y Gasset 1937/2004: 17-19)

7. «Formadas las lenguas en paisajes diferentes y en vista de experiencias distintas, es natural su incongruencia. Es falso, por ejemplo, suponer que el español llama bosque a lo mismo que el alemán llama Wald, y, sin embargo, el diccionario nos dice que Wald significa bosque. Si hubiera humor para ello sería excelente ocasión para intercalar un "aria de bravura» describiendo el bosque de Alemania en contraposición al bosque español. Hago gracia a ustedes de la canción, pero reclamo su resultado : la clara intuición de la enorme diferencia que entre ambas realidades existe.» (Ortega Y Gasset 1937/2004: 23)

8. La référence fournie par Holmes est ici reproduite intégralement: «Hempel, Carl G. Fundamentals of Concept Formation in Empirical Sciences (Chicago: University of Chicago Press, 1967; International Encyclopedia of Social Science [sic], Foundations of the Unity of Sciences, II, Fasc. 7), p. 1.» Nous n’avons pu consulter la référence de Hempel indiquée par Holmes, car elle recèle une erreur, qui se retrouve dans le volume original ainsi que dans la reproduction du texte dans The Translation Studies Reader (Venuti 2004). (Il semble y avoir confusion avec l'International Encyclopedia of Social Sciences, qui est parue en 1968 sous la direction de David L. Sills et Robert K. Merton.) Toury (1995: 3) cite cependant la référence exacte:

Hempel, Carl G. (1952): Fundamentals of Concept Formation in Empirical Sciences. In: Otto Neurath, Rudolf CARnap et Charles Morris. International Encyclopedia of Unified Science, vol. 2, $n^{\circ}$ 7. Chicago: University of Chicago Press.

9. Les représentations en arbre de Porphyre limitées aux deux relations susnommées et unidirectionnellement orientées (à la manière de thésaurus) ont fait l'objet de nombreuses critiques par les chercheurs s'attachant à la représentation des connaissances. On leur préfère généralement les ontologies, qui autorisent des relations variées pouvant être réciproques.

10. Chesterman (2009: 14) fait remarquer que Toury a modifié la carte: «The famous map has circulated in many publications, such as Toury 1995: 10. However, the published version of Holmes' original article (1988) does not actually contain it in diagram form. Curiously, some versions of the figure (such as Toury's) omit the branch on translation policy, which is nevertheless explicitly listed in the article itself.»

11. Nous ne voulons pas entrer ici dans un débat social et politique du financement de la recherche qui s'insinue parfois dans des interstices rhétoriques à l'argumentation angélique (nous renvoyons le lecteur à notre éditorial du numéro 59(1) portant sur l’accès libre aux revues; Vandaele 2014: $1-7)$.

12. Gregor Reisch était originaire de Balingen. Après avoir étudié à Constance et Ingolstadt, il fut admis en 1496 chez les Chartreux de Freiburg-im-Breisgau dont il devint le prieur en 1502. Reisch dirigea la publication des Règles de son ordre, ainsi que de l'œuvre de Saint Jérôme par l'éditeur Johannes Amerbach (Cunningham et Kusukawa 2010: xviii).

13. L'édition d'où est tirée la Philosophiae partitio de la figure 2 est celle qui est accessible par GoogleLivres. Elle porte la date de 1496, date à laquelle on sait que Reisch écrivit le texte à la main. D’après le catalogue dressé par Cunningham et Kusukawa (2010: xxviii) et son titre, cette édition serait celle de 1503 ou 1504, imprimée soit à Freiburg-im-Breisgau, soit à Strasbourg par Johannes Schott, un élève de Reisch. (Cunningham et Kusukawa 2010: ix; xxviii). La Margarita philosophica fut rééditée au moins douze fois au $\mathrm{XVI}^{\mathrm{e}}$ siècle, mais parfois sans autorisation de l'auteur. Le piratage n'a pas attendu l'ère numérique...

ReISCH, Gregor (1496/1503 ou 1504): Margarita philosophica [totius philosophae rationalis, naturalis et moralis principia dialogice duodecim libris complectens]. Freiburg ou Strasbourg: Johannes Schott. Consulté le 05 juin 2015, <https://books.google.ca/books?id=yh5NAAAAcAAJ\&printsec $=$ frontcover $\& \mathrm{dq}=\& \mathrm{hl}=\mathrm{fr} \# \mathrm{v}=$ onepage $\& \mathrm{q}=$ freiburg $\& \mathrm{f}=$ false $>$. 
14. Le réalisme (des universaux) plaide pour une existence en soi de ces derniers (pensée platonicienne idéaliste), tandis que le nominalisme affirme qu'ils sont produits par l'esprit et s'expriment par des noms (pensée aristotélicienne, catégorisation). La querelle réapparaît au $\mathrm{xx}^{\mathrm{e}}$ siècle dans le cadre de la philosophie analytique.

15. La via antiqua (représentée par Thomas d'Aquin et Duns Scotus et le nominalisme, inspirée d'Aristote) et la via moderna (représentée par Guillaume d'Ockham et Grégoire de Rimini et le réalisme, inspirée par Platon).

16. Boèce (Anicius Manilius Severinus Bœthius), philosophe et homme politique romain, est un relais de la philosophie aristotélicienne pour le monde médiéval. Il inspire, entre autres, Thomas d'Aquin. Traducteur d'Aristote et de Platon en latin, il tente de réconcilier leurs positions, mais sera à l'origine de la querelle des universaux.

Bсtтhius, d'Anicius Manlius Severinus (s.d.) De la Trinité - Comment la Trinité est un Dieu et non trois Dieux. Traducteur inconnu. Consulté le 12 juin 2015, <https://fr.wikisource.org/wiki/De_la_ trinité_(Boèce) $>$.

17. Il faut entendre calcul au sens large de projections effectuées à partir d'un point de départ et non pas restreint à un sens arithmétique ou informatique (algorithmes).

18. Le terme a été repris en anglais par Muñoz Martín (2010).

19. Vandaele, Sylvie (2011): Challenging Holmes's Map of Translation Studies: What Research in Specialised Translation Means? Communication non publiée. Congrès de la CIUTI, 21-22 mai 2011, Beijing.

20. En 2007, près d'une cinquantaine de prothèses du genou (opération extrêmement douloureuse) furent réalisées avec une prothèse inadéquate en raison d'une erreur de traduction: l'opération avait été réalisée sans ciment, ce qui entraînait une instabilité permanente de l'articulation. Les patients durent se soumettre à une autre intervention.

Kotlorz, Tanja (10 août 2007) : 47 Berlinern falsches Kniegelenk eingesetzt [47 Berlinois reçoivent une prothèse de genou inadéquate]. Die Weit. Consulté le 15 mai 2015, <http://www.welt.de/ regionales/berlin/article1096822/47-Berlinern-falsches-Kniegelenk-eingesetzt.html>.

\section{RÉFÉRENCES}

Aixelá, Javier Franco (2004): Specialized and Technical Translation, History of Translation and Interpreting. JoSTrans. 1(1):29-49.

Aristote (Ive s. av. J.-C./1959): Éthique à Nicomaque. Paris: J. Vrin.

Aristote (Ive s. av. J.-C./1988/2010): De l'âme. Paris: J. Vrin.

Basalamah, Salah (2012): Un bilan de 40 ans de traductologie - Entrevue avec Alexis Nouss. Global Media Journal - Édition canadienne. 5(1):29-37.

Bassnett, Susan (2012): Translation studies at the cross-roads. Target. 24(2):15-25.

BAstin, Georges L., dir. (2004): L'histoire de la traduction et la traduction de l'histoire (numéro spécial). Meta. 49(3).

Bastin, Georges L. (2005): Sujectivity and rigour. In: Georges L. Bastin et Paul Bandia, dir. Charting the Future of Translation History. Ottawa: Les Presses de l'Universite d'Ottawa, 122-123.

Bensaude-Vincent, Bernadette (2008/2012): «Pur» et «appliqué»: une invention à dépasser. In: Bensaude-Vincent, Bernadette, dir. Matière à penser - Essais d'histoire et de philosophie de la chimie. Paris: Presses universitaires de Paris Ouest/OpenEdition Books.

Bensimon, Paul (1987): Présentation. Palimpsestes. 1:i-iii.

Berman, Antoine (1985a): La traduction comme épreuve de l'étranger. Texte. 4:67-81.

Berman, Antoine (1985b): La traduction et la lettre, ou l'Auberge du lointain. In: Berman, Antoine, dir. Les tours de Babel. Essais sur la traduction. Mauvezin: Éditions Trans-EuropeRepress, 33-150.

Brems, Elke, Meylaerts, Reine et van Doorslaer, Luc (2012): A discipline looking back and looking forward. Target. 24(1):1-14.

Chesterman, Andrew (2004): Translation as an object of research. In: Kittel, Harald, Frank, Armin Paul, Greiner, Norbert et coll., dir. Übersetzung, Translation, Traduction. Berlin: de Gruyter, 93-100.

Chesterman, Andrew (2009): The Name and Nature of Translator Studies. Hermes, Journal of Language and Communication Studies. 43:13-22. 
Chesterman, Andrew et Wagner, Emma (2010): Can Theory Help Translators? A Dialogue Between the Ivory Tower and the Wordface. Londres/New York: Routledge.

Chevrel, Yves, D'hulst, Lieven et Lombez, Christine, dir. (2012): Histoire des traductions en langue française - XIX $X^{e}$ siècle (1815-1914). Lagrasse: Verdier.

Cunningham, Andrew et Kusukawa, Sachiko, dir. (2010): Natural Philosophy Epitomised: A Translation of Books 8-11 of Gregor Reisch's Philosophical Pearl (1503). Surrey: Ashgate.

Dollerup, Cay (2005): Tackling cultural communication in translation. Interculturality and Translation: International Review. 1(1):11-30.

Duris, Pascal (2008): Traduire la science - Hier et aujourd'hui. Pessac: Maison des Sciences de l'Homme d'Aquitaine.

Foz, Clara (2004): Avant-propos - Misère et splendeur de la traduction [Miseria y esplendor de la traducción]. TTR: traduction, terminologie, rédaction. 17(1):13-15.

Froeliger, Nicolas (2010): Le facteur local comme levier d'une traductologie pragmatique. Meta. 55(4):642-660.

GiLe, Daniel (2005) : La traduction: la comprendre, l'apprendre. Paris: Presses Universitaires de France.

GodARD, Barbara (2001): L'Éthique du traduire: Antoine Berman et le «virage éthique» en traduction. TTR: traduction, terminologie, rédaction. 14(2):49-82.

Goffin, Roger (1971): Pour une formation universitaire 'sui generis' du traducteur: Réflexions sur certains aspects méthodologiques et sur la recherche scientifique dans le domaine de la traduction. Meta. 16:57-68.

Holmes, James S., dir. (1971): The nature of translation. Approaches to translation studies. Vol. 1. Paris/La Hague/Bratislava: Mouton/Académie slovaque des sciences.

Holmes, James S. (1972/2004): The Name and Nature of Translation Studies. In: Venuti, Lawrence, dir. The Translation Studies Reader. Londres: Routledge, 180-192.

Holmes, James S. (1988): The Name and Nature of Translation Studies. In: Translated! Papers on Literary Translation and Translation Studies (anthologie posthume). Amsterdam: Rodopi, 67-80.

Kelly, Dorothy (2005) : A Handbook for Translator Trainers. Manchester: St. Jerome Publishers. Kleiber, Georges (2001) : Remarques sur la dénomination. Cahiers de praxématique. 36:21- 41. Ladmiral, Jean-René (2002): Traduire: théorèmes pour la traduction. $2^{\mathrm{e}}$ éd. Coll. Tel. Paris: Gallimard.

Ladmiral, Jean-René (1992): Antoine Berman in memoriam. Translittérature. 2:63-64.

LAKOFF, Georges et JoHnson, Mark (1985/2003): Metaphors we live by [with a new afterwords]. $2^{\mathrm{e}}$ éd. London: The University of Chicago Press.

Lakoff, Georges (1987/1990): Women, Fire, and Dangerous Things - What Categories Reveal About the Mind. Chicago: The University of Chicago Press.

LAmbert, José (1991): Shifts, Oppositions and Goals in Translation Studies: Towards a Genealogy of Concepts. In: van Leuven-Zwart, Kitty et NaAijkens Ton, dir. Translation Studies: The State of the Art. Amsterdam: Rodopi, 25-37.

LEFEVERE, André (1977): Translating Literature: the German Tradition from Luther to Rosenweig. Assen/Amsterdam: Van Gorcum.

LiAnERI, Alexandra (2002): Translation and the Ideology of Culture Reappraising Schleiermacher's Theory of Translation. Current Writing - Text and Reception in Southern Africa. 14(2):2-18.

Malmerbe, Jean-François (1976/2011): La philosophie de Karl Popper et le positivisme logique. Montrél: Liber.

Montalt Resurrecció, Vicent et García Izquierdo, Isabel (2002): Translating into Textual Genres. Lingüística Antverpiensia. 1:135-145.

Montalt Resurrecció, Vicent, Ezpeleta Piorno, Pilar et García Izquierdo, Isabel (2008): The Acquisition of Translation Competence through Textual Genre. Translation Journal. 12(4):s.p. 
Munday, Jeremy (2012): Introducing Translation Studies - Theories and Applications. Londres/ New York: Routledge.

Muñoz Martín, Ricardo (2010): On Paradigms and Cognitive Translatology. In: Shreve, Gregory et Angelone, Erik, dir. Translation and Cognition. Amsterdam/Philadelphie: John Benjamins, 169-187.

Neurath, Otto, Carnap, Rudolf et Hahn, Hans (1973): Wissenschaftliche Weltauffassung: Der Wiener Kreis [The Scientific Conception of the World: The Vienna Circle]. In: Neurath, Otto et Cohen Robert S., dir. Empiricism and Sociology. Dordrecht: Reidel, 299-318.

Newmark, Peter (2004): Non-literary in the Light of Literary Translation. JoSTrans. 1(1):8-13.

Ortega y Gasset, José (1937/2004): Misère et splendeur de la traduction [Miseria y esplendor de la traducción]. TTR: traduction, terminologie, rédaction. 17(1):16-53.

Philips, Denise (2012): Acolytes of Nature: Defining Natural Science in Germany, 1770-1850. Chicago: University of Chicago Press.

Pyм, Anthony (1998): Methods in Translation History. Manchester: St. Jerome.

Pyм, Anthony (2009): Empirisme et mauvaise philosophie en traductologie [On empiricism and bad philosophy in translation studies]. In: Milliaressi, Tatiana, dir. De la linguistique à la traductologie - interpréter/traduire. Villeneuve d'Ascq: Presses universitaires du Septentrion, 53-69.

Rogers, Margaret (2015): Specialised Translation: Shedding the 'Non-iterary' Tag. Basingstoke/ New York: Palgrave Macmillan.

Rovira-Esteva, Sara, Orero, Pilar et Aixelá, Javier Franco (2015): Bibliometric and bibliographical research in Translation Studies. In: Rovira-Esteva, Sara, Orero Pilar et Aixelá Javier Franco, dir. Bibliometric and Bibliographical Research in Translation Studies. 23, 159-160.

SCARPA, Federica (2010): La traduction spécialisée - Une approche professionnelle à l'enseignement de la traduction. Traduit par Marco Fiola. Ottawa: Les Presses de l'Université d'Ottawa.

Schleiermacher, Friedrich Daniel Ernst (1799/1944): Discours sur la religion [Über die Religion]. Paris: Aubier.

Schleiermacher, Friedrich Daniel Ernst (1813/1838): Ueber die verschiedenen Methoden des Uebersezens. In: Friedrich Schleiermacher's sämmtliche Werke, Dritte Abteilung: Zur Philosophie, Zweitter Band. Berlin: Reimers, 207-245.

Schleiermacher, Friedrich Daniel Ernst (1813/1985/1999): Des différentes méthodes du traduire/Ueber die verschiedenen Methoden des Uebersezens. Paris: Éditions du Seuil.

Schleiermacher, Friedrich Daniel Ernst (1978/2000): Sobre los diferentes métodos de traducir - Traducción y comentarios de Valentín García Yebra. Madrid: Gredos.

Shreve, Gregory et Angelone, Erik, dir. (2010): Translation and Cognition. Amsterdam/ Philadelphie: John Benjamins.

Snell-Hornby, Mary (1991): Translation Studies - Art, Science or Utopia? In: van LeuvenZwart, Kitty et NaAijkens Ton, dir. Translation Studies: The State of the Art. Amsterdam: Rodopi, 13-23.

Thouard, Denis (2003): Qu'est-ce que les Lumières pour le premier romantisme? Chimie, Witz, maximes et fragments: Friedrich Schlegel et Chamfort. texto! Textes \& Cultures.

Toury, Gideon (1995/2012): Descriptive Translation Studies - and Beyond (Revised edition). Amsterdam/Philadelphie: John Benjamins.

van Doorslaer, Luc (2007): Risking the conceptual maps. Target. 19(2):217-233.

VANDAele, Sylvie (2000) : Métaphores conceptuelles et traduction biomédicale. In: MéjRI, Sahla, Baccouche, Taïeb, Clas, André et Gross, Gaston, dir. La traduction: théorie et pratiques. Tunis: Publications de l'ENS, 393-404.

Vandaele, Sylvie (2006a): Le masque de la banalité: le champ sémantique de l'évaluation thérapeutique 1 - La pharmacologie classique. Pharmaterm. 17(3):1-4.

Vandaele, Sylvie (2006b): Le masque de la banalité: le champ sémantique de l'évaluation thérapeutique 2 - La pharmacognosie. Pharmaterm. 17(4):1-4. 
VANDAELE, Sylvie (2007): Quelques repères épistémologiques pour une approche cognitive de la traduction spécialisée - Application à la biomédecine. Meta. 52(1):129-145.

VAndAele, Sylvie (2008): Les parties constitutives du cerveau: aimez-vous le cube de Rubik? Pharmaterm. 19(3):1-6.

VANDAELE, Sylvie (2009): Les modes de conceptualisation du vivant: une approche linguistique. In: Boucher, François-Emmanuel, David, Sylvain et Przychodzen, Janusz, dir. Pour ou contre la métaphore? Pouvoir, histoire, savoir et poétique. Paris: L'Harmattan, 187-207.

VAndaele, Sylvie (2013): Du néologisme au domaine du savoir: les cas des sciences de la vie. Neologica. 7:157-185.

VANDAele, Sylvie (2014): Éditorial. Meta. 59(1):1-7.

VANDAELE, Sylvie et RAfFo, Mariana (2008): Significations lexicales et notionnelles dans les domaines de spécialité. In: MAniez, François et Dury, Pascaline, dir. Lexicologie et terminologie: histoire de mots. Lyon: Travaux du CRTT, 249-268.

VAndaele, Sylvie et Gingras-Harvey, Mariane (2013): Les nomenclatures anatomiques: histoire et traduction.Panace@.XIV(38):222-234.

Vandaele, Sylvie et Gendron-Pontbriand, Eve-Marie (2014): Des «vilaines infidèles» à la postérité: traduction et retraduction de Charles Darwin. In: Pinilla Martinez, Julia et LÉPINETTE Brigitte, dir. Traducción y difusión de la ciencia y de la técnica en España (s. XVIXIX). València: Universitat de València, 249-275.

Vandaele, Sylvie, Gendron-Pontbriand, Eve-Marie et Olivier-Bonfils, Delphine (2013): Représentations des espèces moléculaires et syntagmes nominaux en français. Panace@. XIV(38):253-262.

VANDE WALle, Jean-Marie (2007): Le traducteur et la technique, un couple infernal. In: Maria Teresa Musacchio et Geneviève Henrot Sostero, dir. Tradurre: professione e formazione. Padoue: Cleup, 31-44.

VICtORri, Bernard (1994) : La construction dynamique du sens. In: Michèle Porte, dir. Passions des formes - À René Thom. St Cloud: ENS Éditions Fontenay, 733-747.

WALtER, Henriette (2006) : Quelques réflexions sur les formes empruntées hybrides. Revue belge de philologie et d'histoire. 84(3):665-671.

Vorms, Marion (2009): Théories, modes d'emploi: une perspective cognitive sur l'activité théorique dans les sciences empiriques. Thèse de doctorat. Paris: Université PanthéonSorbonne - Paris I. Consulté le 10 mai 2015, <https://tel.archives-ouvertes.fr/tel-00462403>.

Wuillème, Tanguy (2011): José Ortega y Gasset, La révolte des masses. Questions de communication. 19:s.p.

Sylvie Vandaele a été directrice de Meta pendant six ans, de 2008 à 2014. Elle détient une double formation en sciences et en traduction. Après un diplôme de doctorat en pharmacie (Université de Marseille, 1982) et un doctorat ès sciences de la vie (pharmacologie moléculaire; Université de Nice, 1987), elle mène des recherches en neurosciences à l'université Harvard, à l'université McGill et à l'Université de Montréal jusqu'en 1995. Après une reconversion en traduction et la constitution d'un cabinet indépendant, elle revient à la vie universitaire en 2000 au sein du Département de linguistique et de traduction de l'Université de Montréal. Elle y enseigne la traduction dans les domaines de la médecine et des sciences de la vie (biotechnologies) et elle dirige le groupe de recherche BiomeTTico. Son intérêt de recherche principal concerne les modes de conceptualisation métaphorique en sciences, envisagés dans une perspective synchronique autant que diachronique, c'est-à-dire sous l'angle de l'histoire des sciences, que complètent des recherches terminologiques ainsi qu'une réflexion continue sur l'enseignement et sur la pratique professionnelle. Traductrice et terminologue agréée, elle contribue régulièrement à la formation continue des professionnels dans le cadre de l'Ordre des traducteurs, terminologiques et interprètes agréés du Québec. Enfin, elle est membre de l'Association canadienne de traductologie et de l'American Translator Association, ainsi que de la History of Science Society (É.-U.) et de la Société française d'histoire de la médecine. 